\title{
Gene network analysis to determine the effect of hypoxia-associated genes on brain damages and tumorigenesis using an avian model
}

Hamed Kharrati-Koopaee ${ }^{1,2^{*}}$, Esmaeil Ebrahimie ${ }^{1,3,4}$, Mohammad Dadpasand ${ }^{5 *}$, Ali Niazi ${ }^{1}$, Rugang Tian $^{6}$ and Ali Esmailizadeh ${ }^{*}$

\begin{abstract}
Background: Hypoxia refers to the condition of low oxygen pressure in the atmosphere and characterization of response to hypoxia as a biological complex puzzle, is challenging. Previously, we carried out a comparative genomic study by whole genome resequencing of highland and lowland Iranian native chickens to identify genomic variants associated with hypoxia conditions. Based on our previous findings, we used chicken as a model and the identified hypoxia-associated genes were converted to human's orthologs genes to construct the informative gene network. The main goal of this study was to visualize the features of diseases due to hypoxiaassociated genes by gene network analysis.

Results: It was found that hypoxia-associated genes contained several gene networks of disorders such as Parkinson, Alzheimer, cardiomyopathy, drug toxicity, and cancers. We found that biological pathways are involved in mitochondrion dysfunctions including peroxynitrous acid production denoted in brain injuries. Lewy body and neuromelanin were reported as key symptoms in Parkinson disease. Furthermore, calmodulin, and amyloid precursor protein were detected as leader proteins in Alzheimer's diseases. Dexamethasone was reported as the candidate toxic drug under the hypoxia condition that implicates diabetes, osteoporosis, and neurotoxicity. Our results suggested DNA damages caused by the high doses of UV radiation in high-altitude conditions, were associated with breast cancer, ovarian cancer, and colorectal cancer.

Conclusions: Our results showed that hypoxia-associated genes were enriched in several gene networks of disorders including Parkinson, Alzheimer, cardiomyopathy, drug toxicity, and different types of cancers. Furthermore, we suggested, UV radiation and low oxygen conditions in high-altitude regions may be responsible for the variety of human diseases.
\end{abstract}

Keywords: Hypoxia, Brain damages, Gene network, Tumorigenesis

\footnotetext{
*Correspondence: h.kharrati.ko@gmail.com; Dadpasand@shirazu.ac.ir; aliesmaili@uk.ac.ir

${ }^{1}$ Institute of Biotechnology, Shiraz University, Shiraz, Iran

${ }^{5}$ Department of Animal Science, School of Agriculture, Shiraz University,

Shiraz, Iran

${ }^{2}$ Department of Animal Science, Faculty of Agriculture, Shahid Bahonar

University of Kerman, Kerman, Iran

Full list of author information is available at the end of the article
}

\section{Springer Open}

( ) The Author(s). 2021 Open Access This article is licensed under a Creative Commons Attribution 4.0 International License, which permits use, sharing, adaptation, distribution and reproduction in any medium or format, as long as you give appropriate credit to the original author(s) and the source, provide a link to the Creative Commons licence, and indicate if changes were made. The images or other third party material in this article are included in the article's Creative Commons licence, unless indicated otherwise in a credit line to the material. If material is not included in the article's Creative Commons licence and your intended use is not permitted by statutory regulation or exceeds the permitted use, you will need to obtain permission directly from the copyright holder. To view a copy of this licence, visit http://creativecommons.org/licenses/by/4.0/. 


\section{Background}

Hypoxia refers to the condition of low oxygen pressure in atmosphere, and is also considered as one of the most important factors that can impact on many biological pathways of cells [52]. Thus, organisms must adapt to the low oxygen environment to increase the chance of survival [66]. It has been demonstrated that there is close association between abnormal level of oxygen and human's disorders and hypoxia is known as the common symptom among human diseases such as cancers, cardiovascular, heart failure, ischemia, cerebral edema, and diabetes [26, 71, 82, 110, 111, 119].

Many studies aimed to explain the role of hypoxia in diseases and disorders. As an example, in high-altitude conditions, high doses of UV (ultraviolet) radiation lead to the DNA damage, cell apoptosis, skin cancer, and tissue injuries in mammals [26, 71, 82, 97, 108, 119]. In addition, the low oxygen concentration in high-altitude conditions is another risk factor for diseases [70]. The lack of normal oxygen level leads to the inadequate mitochondrial metabolism, and also plays a critical role in cell survival [41]. Therefore, any alteration or dysfunction in mitochondrial activity can lead to irreparable injuries of the body's organs, especially in the central nervous system [15], because the brain is considered as the most sensitive organ to the consumption of oxygen and energy.

In this way, cerebral anoxia induces the neuron cell death and apoptosis, which will lead to hypoxic brain injury including Alzheimer, Huntington, and Parkinson $[100,116]$. It should be noted that numerous investigations have attempted to identify candidate genes and molecular mechanisms involved in the process of adaptation to hypoxia and incidence of disorders; however, this process contains complex biological regulatory pathways, leader proteins, and different gene expression patterns [53]. Therefore, there is no evidence for integrated response to hypoxic stress in humans [41].

The network analysis of the hypoxia-regulated proteins can provide new insight for a better understanding of the molecular mechanisms of the adaptation to anaerobic stress and predict roles of key proteins in human diseases [52].

Animal models have been widely used in order to explore diseases and provide novel insights into the mechanisms of human diseases. Although biological pathways of animals do not conform to humans perfectly, many treatment systems and drugs were developed using animal models [16].

Many of the features of avian biology and organization of the chicken genome make it an ideal model organism for phylogenetics and embryology, along with applications in agriculture and medicine $[11,25,28]$. Since the sequencing of the genome and the development of high- throughput tools for the exploration of functional elements of the genome, the chicken has reached model organism status. Functional genomics and computational analyses in chickens provide powerful tools in order to understand the function and regulation of genes and obtaining new insight into the evolution of gene families in birds and other organisms [18].

In this study, based on the chicken hypoxia-associated genes involved in response to hypoxia [51] the biological network analysis was investigated to clarify the roles of key candidate genes in the human hypoxia-related diseases.

\section{Methods}

The current study was founded based on the outcomes of our pervious investigation which were planned to identified hypoxia-associated genes in native chickens. Previously, we carried out a comparative population genomic study by whole genome resequencing of highland and lowland Iranian native chickens to identify genomic variants associated with hypoxia conditions [51]. In this way, 80 hypoxia-associated candidate genes were obtained for gene network analysis. Here, to avoid repetition, the summary of material and method was described. Supplementary file S1 provides the complete details of sampling and analyzing data.

\section{Blood sampling, whole genome sequencing, and phenotypic data}

Briefly, blood samples were collected from ten native chickens in Isfahan (highland, altitude $=2087 \mathrm{~m}$, five samples) and Sari (lowland, altitude $=54 \mathrm{~m}$, five samples) provinces in Iran. Total genomic DNA was isolated from the whole blood and whole genome was sequenced using Hiseq2000 platform and the length of the provided paired-end short reads was $125 \mathrm{bp}$. The raw genome resequencing reads were deposited in the browser of chickenSD database (http://bigd.big.ac.cn/chickensd/). In order to classify the highland and lowland chickens phenotypically, 24 quantitative traits were recorded for principle component analysis (PCA) and discriminate analysis in Minitab software (version: 17). The summary of recorded traits was included body weight (gr), neck length, body size (between waist and pectoral circumference), shank length, body size (between waist and abdominal circumference), wing length, tail length, femur length, crown length, crown height, back cape length, body size (between pectoral and cloaca circumference), head height (height from head to floor), femur diameter and shank diameter. To reduce measurement error, all chickens were matured while recording. It should be noted that the same recording protocol was also used and all chickens were matured while recording. 
Supplementary file S1 gives more detail about phenotypic data analysis.

\section{Measurement of UV radiation and atmosphere pressure} Atmosphere pressure and UV dose are different in highland and lowland regions. The measurement of UV dose is associated with environmental conditions such as earth-to-sun distance, absorption by atmospheric gases, air pollutions, and also the amount of atmosphere pressure depends on temperature and humidity [50, 85]. Therefore, in order to show the UV dose and atmosphere pressure in selected highland and lowland regions, the recent annual average was reported for Isfahan (highland; UV: $3228.83 \pm 51.12\left(\mathrm{j} / \mathrm{m}^{2}\right)$, atmosphere pressure: $760.63 \pm 12.19(\mathrm{mmHg})$ ) and Sari (lowland; UV: $2569.60 \pm 41.87\left(\mathrm{j} / \mathrm{m}^{2}\right)$, atmosphere pressure: $1015.12 \pm$ 16.74 (mmHg)) (www.weather.ir).

\section{Quality control, trimming, and mapping}

The function of quality control in CLC Genomic Workbench (8.5.1) was applied by the following parameters for each sample: length distribution, GC content, ambiguous base content, Phred score, nucleotide contribution, enrich 5 mers, and duplicate sequences [22]. The adaptor sequences were removed by Illumina Company; thereby, trimming was carried out based on other parameters. The reference genome and annotations were downloaded from the Ensembl database. Annotations included gene annotations and variants (ftp://ftp.ensembl. org/pub/relase-84/fasta/gallus_gallus). Mapping was performed against the reference genome. Briefly, mapping parameters were included mismatch cost $=2$, insertion cost $=3$, deletion cost $=3$, length fraction $=0.7$, similarity fraction $=0.8[69]$. More details were provided in supplementary file S1.

\section{Genetic variants detection and gene ontology enrichment analysis}

The variant detection algorithm was run by CLC genomics workbench (8.5.1). The ploidy level was fixed in chickens $(2 n=78)$. Therefore, fixed ploidy algorithm was applied for variant calling based on the following parameters: minimum count $=2$, minimum frequency $(\%)$ $=30$, base quality filter $=$ yes, neighborhood radius $=15$, minimum central quality $=30$, minimum neighborhood quality $=25[22,23]$.

After variants calling, variations of highland chickens were compared to the reads of lowland chickens as a control tool in order to remove the common variation between lowland and highland samples. The file of gene ontology (GO) association, which included gene names and associated gene ontology terms, was downloaded from the gene ontology consortium (http:// geneontology.org/) and imported to CLC Genomic
Workbench (8.5.1). The output of amino acid change analysis was applied in order to analyze the gene ontology enrichment of the biological process, molecular function, and cellular component. The significance level of GO analysis was determined to be 0.01 . Finally, based on the results of $\mathrm{GO}$ analysis, candidate genes associated with significant GO term $(P \leq 0.01)$ were used in the gene network analysis [51]. The lists of selected genes are presented in Table S1. The graphic abstract of genetic variants detection and gene network analyses are briefly depicted in Fig. 1.

\section{Gene network analysis}

Gene network analysis was performed based on hypoxiaassociated candidate genes which were reported by comparative genomic study between highland and lowland chickens [51]. Unlike, chicken's genes a lot of information of human's genes are available in the database of Studio pathway software web (Elsevier). Therefore, the identified chicken Ensembl IDs were converted to human orthologs by BioMart tool in Ensembl database (https://asia.ensembl.org/) in order to build an informative gene network (Table S1) [22]. BioMart tool provides an easy-to-use web-based tool that allows extraction of data without any programming knowledge or understanding of the underlying database structure. Gene network analysis was carried out by Pathway Studio web (Elsevier), with the purpose of identifying gene network and upstream neighbors network for diseases based on parameters of Min overlap $=2$, and $P$ value $<$ 0.05 [75].

\section{Results}

The complete results of genetic variants detection that were published in our investigation [51] are provided in supplementary file S1. However, the summary outcomes of the previous study describe here in order to clarify the identification of hypoxia-associated genes.

A total of more than 20 million variations were generated, including single nucleotide variant (SNV), multi nucleotide variant (MNV), insertion, deletion, and replacement. In order to remove the common variants between highland and lowland chickens, total variants of those samples were compared. A total of 97,610 and 17, 024 genetic variants were detected for both males and females as the differential variants between highland and lowland chickens, respectively. The results of gene ontology analysis indicated that the most frequent significant GO terms belonged to DNA repair, histone binding, pericardium morphogenesis, thalamus development, and immune response. Finally, 80 candidate genes, associated with significant $\mathrm{GO}$ terms $(P \leq 0.01)$ were selected for gene network analysis (Table S1). 


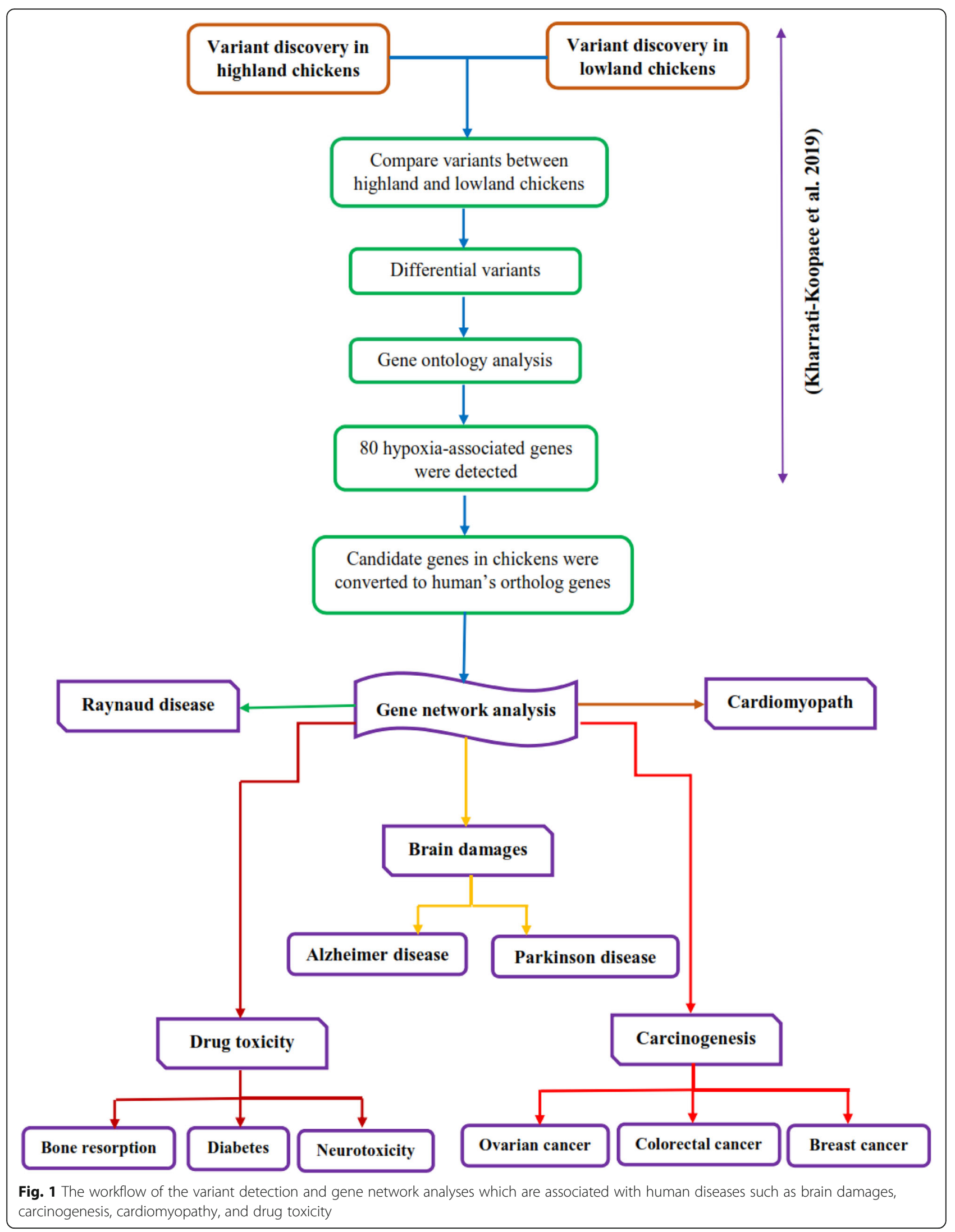


The outcomes phenotypic data analysis indicated that lowland and highland chickens can be classified into two separated groups, phenotypically. The accuracy was estimated to be $75 \%$ (Tables S2-S3).

\section{Gene network analysis}

The nomenclature of network figures is given in Table 1, and the summary results of gene network building are shown in Table 2.
Table 2 shows results of gene network construction by hypoxia-associated genes. "Total gene in database" indicates the count of all available candidate genes for diseases in the database of Pathway Studio software. As an example, 104 candidate genes are available in the database of Pathway Studio for Parkinson disease. "Total genes in network" shows the count of genes that were constructed the network. For instance, 14 candidate genes contributed to network building for Parkinson disease. In the current

Table 1 The legend of entities and relations types for gene network analysis

\begin{tabular}{|c|c|c|c|}
\hline Entities and relations types & Description & Entities type & Description \\
\hline & Disease & & Cell process \\
\hline בَ. & Negative regulation & & Transcription factor \\
\hline 9 & Positive regulation & हs? & Membrane plasma \\
\hline 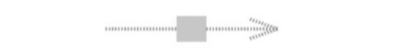 & Regulation (Unknown) & & Mitochondria \\
\hline 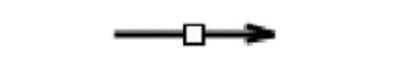 & Chemical reaction & & Nucleolus \\
\hline P & Direct regulation & & Golgi system \\
\hline & Protein modification & & Treatment \\
\hline & Binding & & Small molecule \\
\hline$>$ & Expression & & Protein complex \\
\hline & Promoter binding & & Functional class \\
\hline & Genetic change & & Protein ligand \\
\hline & Protein kinase & & Protein Transporter \\
\hline & Protein & & Receptor \\
\hline
\end{tabular}




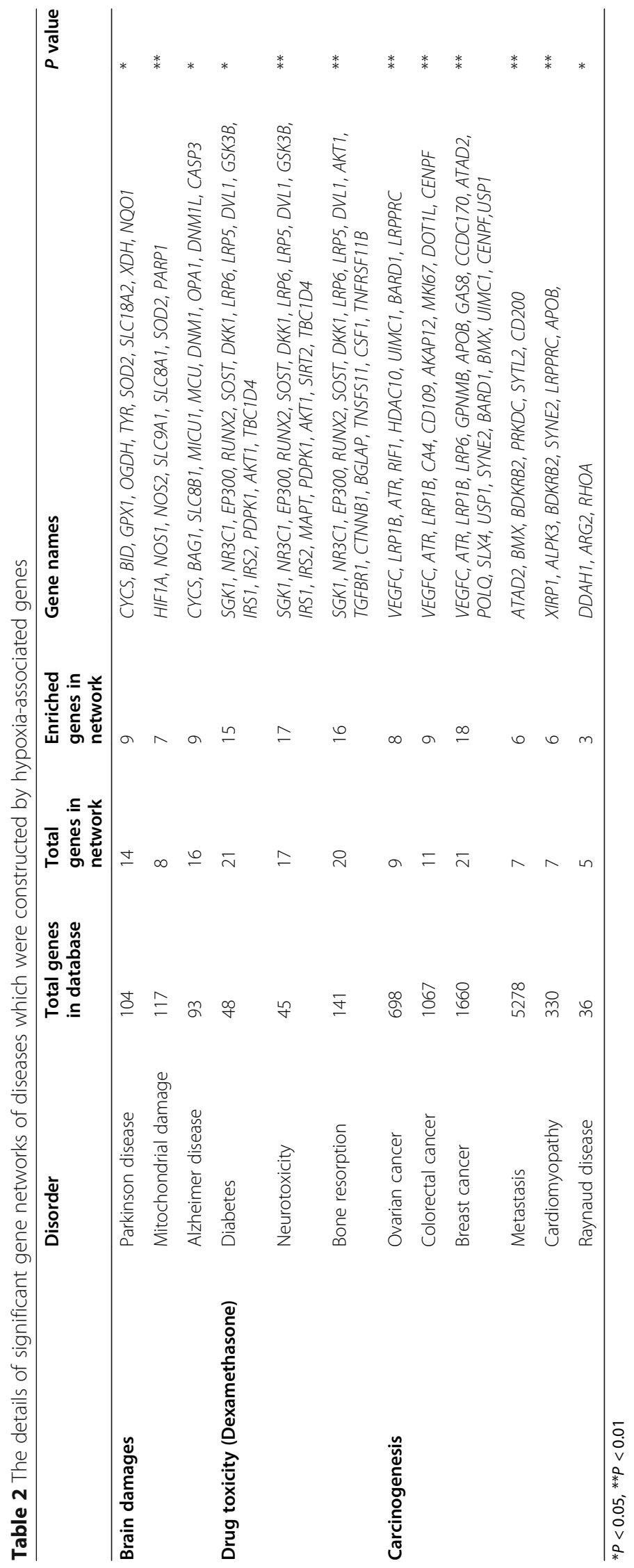


study, 80 hypoxia-associated genes were used for network analysis; therefore, "Enriched genes in network" shows how many of 80 hypoxia-associated genes were enriched in network. For Parkinson disease, 9 candidate genes which we reported as hypoxia-associated genes were enriched in the network.

\section{Brain damages}

Hypoxic brain injuries occur when the brain receives less oxygen than its requirements. Generally, the brain needs the continuous supply of oxygen for its survival and uses $20 \%$ of the body's oxygen intake. Mitochondrion plays an important role in cell respiration and ATP production, while there is a close relationship between mitochondrial oxygen consumptions, neuronal activity, and aerobic metabolisms. Therefore, any interruption of cell respiration can lead to brain injuries [48].
Our findings suggested that there was an association between hypoxia and mitochondrion functions such as glucose metabolism, ATP production, respiratory chain, and Tricarboxylic acid cycle (Fig. 2). Hypoxia, which is considered as an environmental stress, can reduce the cells' $\mathrm{pH}$ level; thus, it will lead to the increased lactate. More importantly, it is proved that the transports process in plasma membrane depends on cellular $\mathrm{pH}$. Therefore, the level of $\mathrm{Ca}^{2+}$ increases in cytoplasm. Figure 2 shows that $\mathrm{Ca}^{2+}$ can lead two processes in the cell. First, it enhances MPTP complex (mitochondrial permeability transition pore) and causes the ATP depletion, mitochondria damage, and apoptosis. Additionally, it was found that MPTP has a regulatory role in ubiquinol-cytochrome $\mathrm{c}$ reductase and NADH dehydrogenase activities, and leads to DNA degradation which itself results in the superoxide and peroxynitrous acid productions. Second, $\mathrm{Ca}^{2+}$ has a

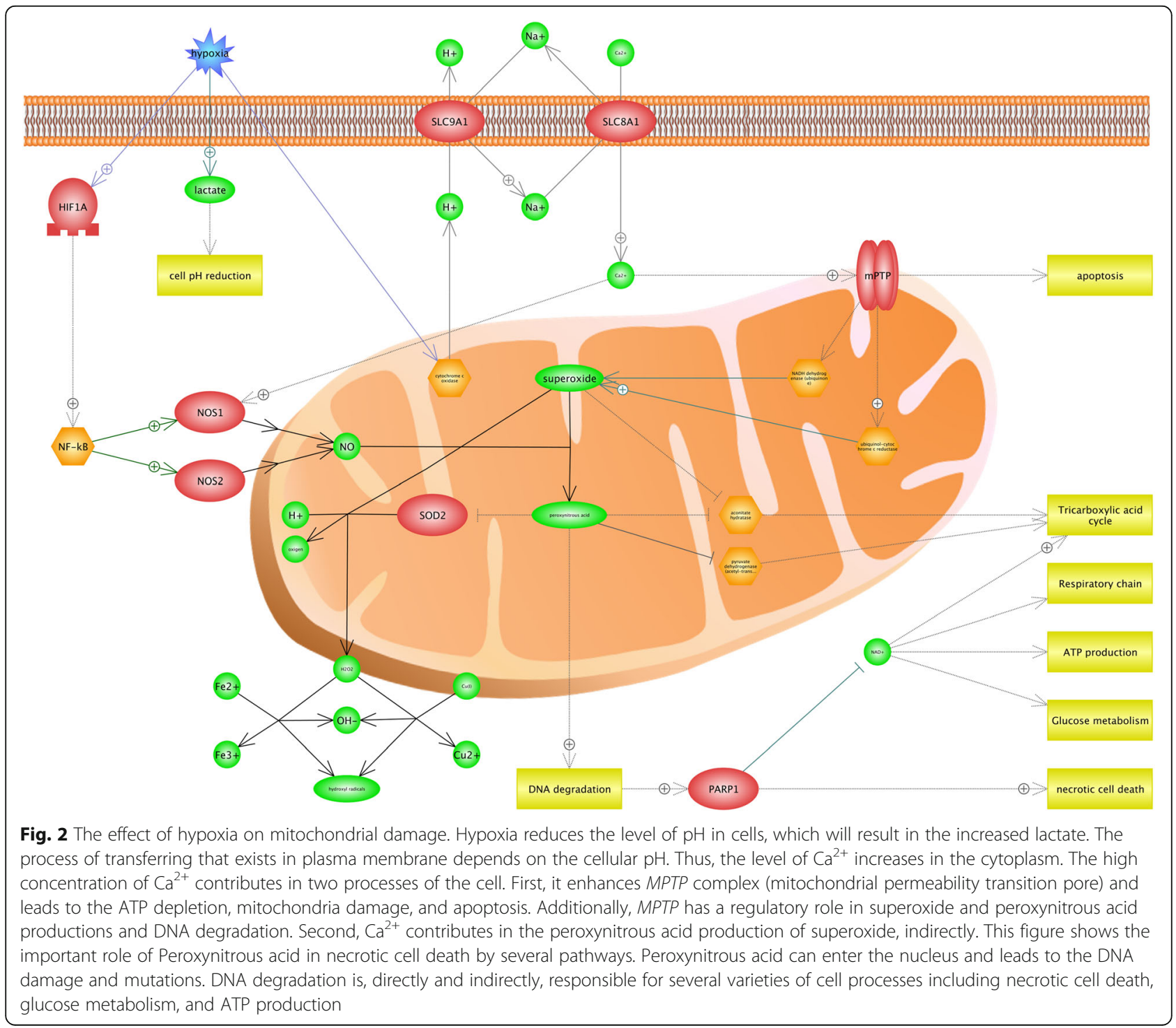


positive effect on NOS1 (Nitric Oxide Synthase 1) and contributes in peroxynitrous acid production from superoxide, indirectly. We found that hypoxia induces the HIF1A (hypoxia-inducible factor 1-alpha) transcription factor and also contributes in the peroxynitrous acid production by activating NOS1 and NOS2 (Nitric Oxide Synthase 2), indirectly. It is observed that hypoxia involves in the process of producing peroxynitrous acid and necrotic cell death by applying several approaches (Fig. 2). Peroxynitrous acid is known as a potent oxidant that reacts with many macro molecules including proteins and lipids. Moreover, peroxynitrous acid can enter the nucleus and cause the DNA damage and mutations [36]. DNA degradation is both directly and indirectly responsible for several varieties of cell processes including necrotic cell death, glucose metabolism, and ATP production. We also found that hydroxyl radicals are generated in cell by the key roles of peroxynitrous acid, superoxide, and SOD2 proteins. It was observed that hydroxyl radicals indicate the apoptosis and cell damages [88].

\section{Parkinson disease}

Parkinson disease (PD) is defined as a neurodegenerative disorder impacts on movements. It is recognized that the incidence of PD has overshadowed; however, several risk factors including age, family history, pesticide exposure, and environmental chemicals were suggested for PD [4]. Substantia nigra, as a long nucleus, is the most important part of the midbrain that plays a critical role in movements of the body. Figure 3 shows that superoxide contributes in neuron apoptosis in the Substantia nigra; it is also found that the Superoxide can lead to oxidative stress and DNA damage by increasing ROS (Reactive oxygen species). Results show that ROS are known as normal forms of oxygen in the process of metabolism; however, the abnormal levels of ROS contribute in the cell damage. Figure 3 illustrates that the CYCS protein can lead to neuron apoptosis. CYCS gene encodes the cytochrome c, which is a member of the electron transport chain in the mitochondrion. Cytochrome c, which is recognized as the electron carrier between the cytochrome $\mathrm{c} 1$ subunit of cytochrome reductase and cytochrome oxidase complex, has a very significant role; however, releasing cytochrome $\mathrm{c}$ into the cytosol can lead to the activation of the apoptosis process in the cell (UniProtKB - P99999). Lewy bodies are well-known as the main symptom of PD [109]. Lewy bodies are associated with unusual deposits of proteins in Substantia nigra. It is believed that $\alpha$-synuclein ( $\alpha$-Syn or SNCA) contributes in the lewy body formation and clumps of $\alpha$ Syn also have a significant role in PD. We found that neuromelanin is considered as another important marker of PD (Fig. 3). Neuromelanins are known as complex structures that contain granules of melanin polymer; however, they are actually dark pigments that are deposited with aging in substantia nigra [91]. It is reported that the loss of Neuromelanin could be considered as an effective symptom of PD. Interestingly, it is suggested that there is a close association between the degeneration of dopamine neuron and high amounts of Neuromelanin. Dopaminergic neurons are recognized as the most significant sources of dopamine in brain, and their dysfunctions are also associated with different types of neurological disorders, especially PD $[2,37,120]$. We also found that rotenone inhibits mitochondrion respiratory chain complex, and induces cell damages. Rotenone is known as a natural plant toxin produced by tropical plants and has been used for more than 150 years in the world because of its insecticide properties. It is proved that levels of rotenone toxicity vary in different animals. For example, it is highly toxicant for aquatic organisms, while has a low degree of toxicity for birds and mammals [61]. It is recognized that rotenone, which is considered as an oxygen sensor, is a key component of hypoxia sensing [103].

\section{Alzheimer disease}

Alzheimer's disease (AD) is related to the central nervous system that impacts on the memory and leads to the cognitive decline [27, 92]. Results indicated that there are two main complexes including TOM (translocase of the outer mitochondrial membrane) and MPTP, which play a critical role in AD (Fig. 4). TOM complex is well-known as the protein translocator of the outer membrane of mitochondrion. Most of the mitochondrion proteins are produced in the cytoplasm and are imported to the mitochondrion by the TOM complex [39]. Figure 4 shows that APP (amyloid precursor protein) inhibits the TOM complex and leads to the mitochondrial damage. We found that there are associations between APP, OPA1, DNM1L, which contribute in the mitochondrion fusion and fission. However, their accurate role in mitochondrial dynamics remains to be determined [40]. It is believed that mitochondrion is dynamic organelle in cells and is also able of fission/ fusion. In fact, fission/fusion is related to the quality control of mitochondrion. Therefore, it is recognized that any alteration in mitochondrial fusion/fission and related proteins contribute in $\mathrm{AD}$ and $\mathrm{PD}$ disorders $[1,44]$. The accumulation of $\beta$-amyloid peptide $(A \beta)$ is considered as one of the hallmark features of $\mathrm{AD}$, and is produced by the degeneration of protein tau; however, it contributes in the process of regulating synaptic scaling and synaptic vesicle release, physiologically [73]. Based on our results mitochondrial calcium is overloaded by $\mathrm{A} \beta$ and MCU (mitochondrial calcium uniporter). $\mathrm{MCU}$ is located on 


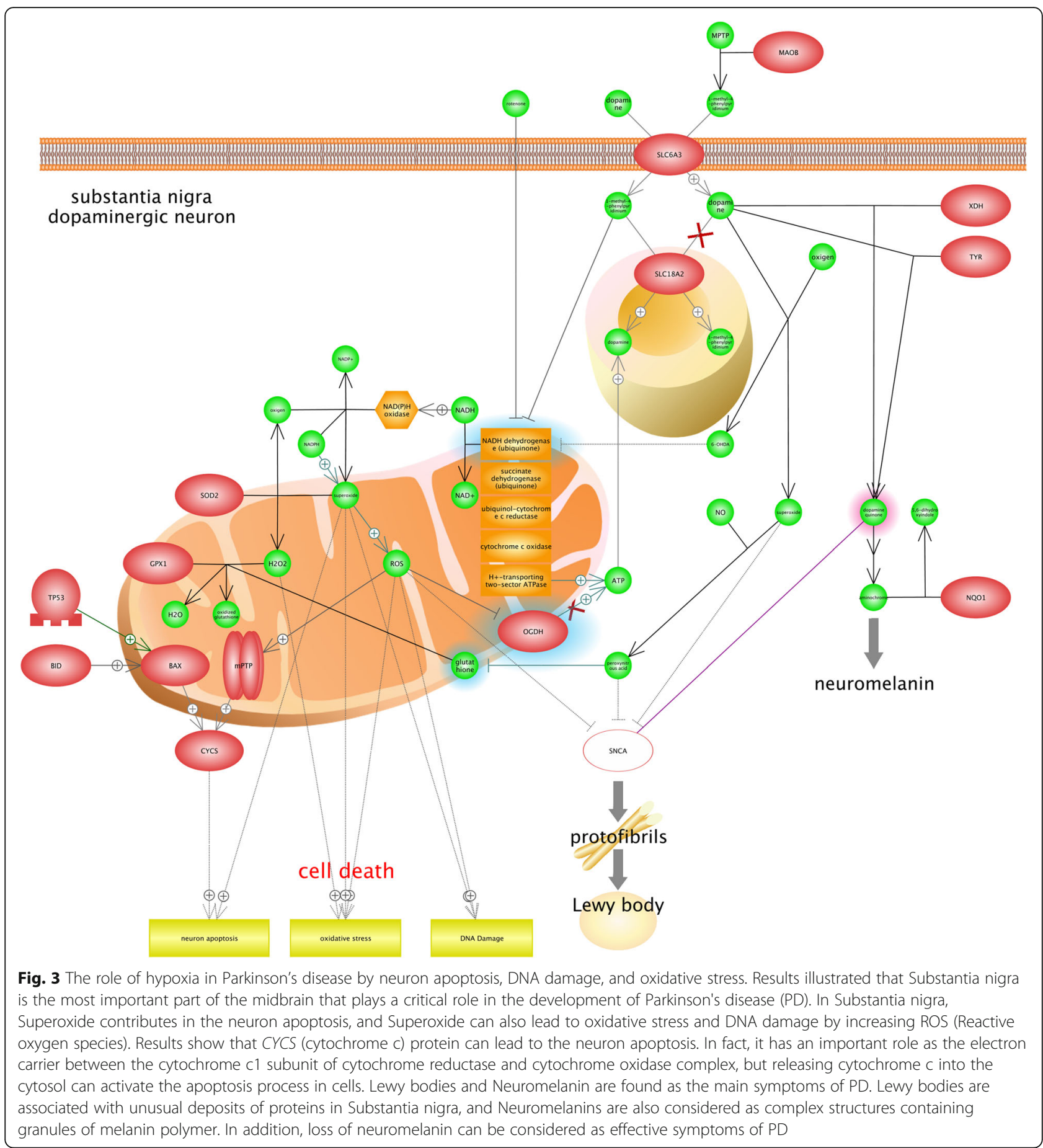

mitochondrial inner membrane and involves the calcium uptake (Uniprot: Q8NE86). According to Fig. 4, there is an association between mitochondrial calcium overload and MPTP activity. MPTP is related to mitochondrial dysfunction because it is opened in the inner mitochondrial membrane; we also found that molecules of $<1.5 \mathrm{KDa}$, including protons, could be transferred to mitochondria and lead to the ATP depletion and mitochondria damage in this situation [38]. Calmodulin is known as a calcium-binding protein involves in the process of regulating different protein targets' multitude; therefore, it impacts on many cellular functions. Calmodulin is considered as a specific biomarker in AD; thus, blood's Calmodulin levels are increased significantly in AD cells, but it is not reported in other neurodegenerative disorders [29]. Additionally, it is demonstrated that calmodulin- 


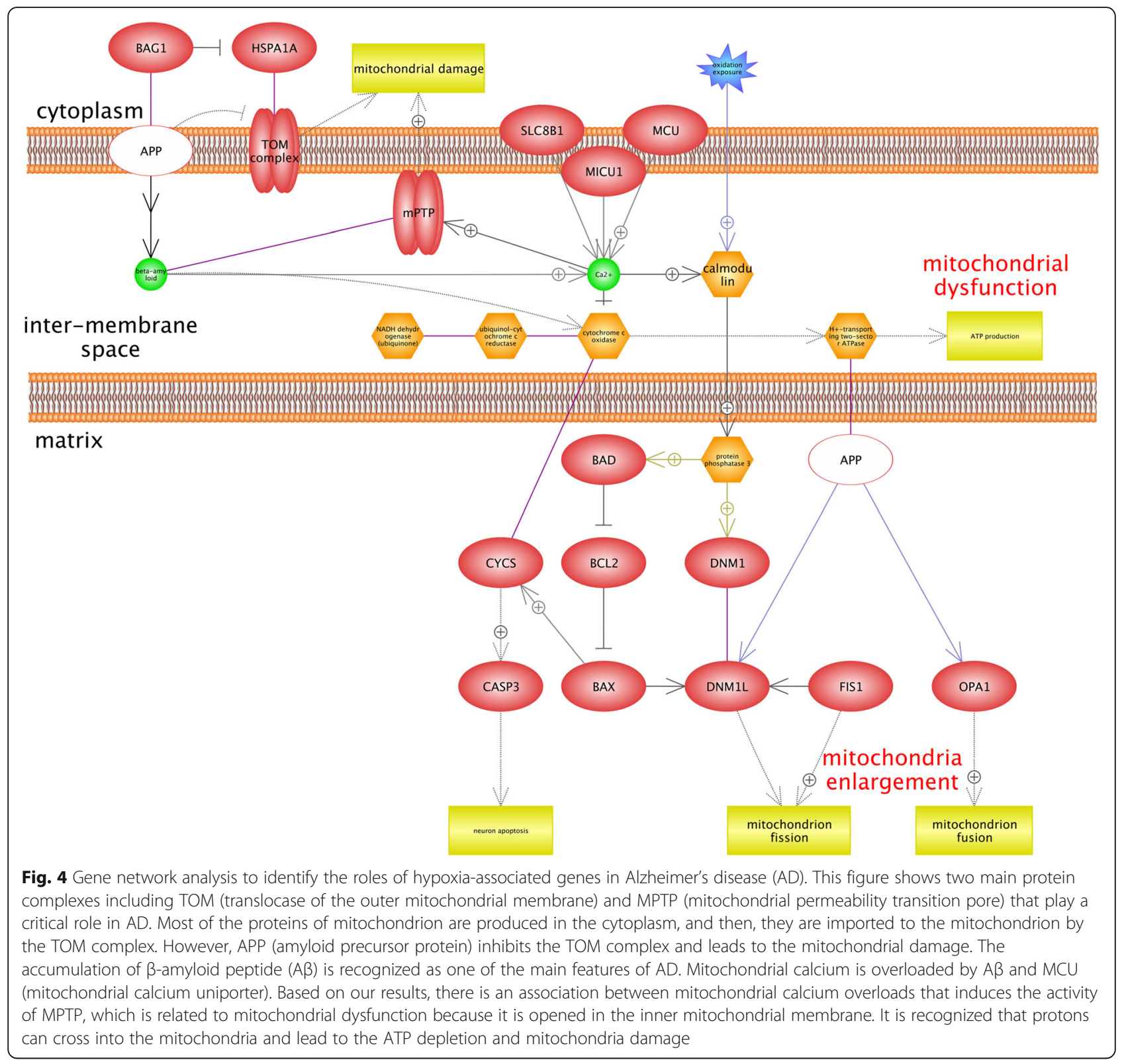

binding proteins play a significant role in the formation of $\mathrm{A} \beta[72]$.

\section{Drug toxicity}

Many pathways of drug metabolisms such as oxidation, sulfation, and acetylation depend on the oxygen availability; thereby, the investigation of drugs and their metabolism pathways can provide beneficial information about optimal drug therapy and avoidance of drugs' side effects in plateau regions [57]. Results of this study showed that Dexamethasone under hypoxia condition induces diabetes, osteoporosis, and neurotoxicity. Dexamethasone is a corticosteroid that prevents the release of substances in the body that cause inflammation. We found that similar networks were constructed for diabetes and neurotoxicity (Figs. 5 and 6). In the first step, dexamethasone induces the transcription factor (TF) NR3C1 (Nuclear Receptor Subfamily 3 group $C$ member 1 ). It has been demonstrated that the NR3C1 gene plays two critical roles. First, it encodes glucocorticoid receptor as a transcription factor that binds to glucocorticoid response elements in the promoters of glucocorticoid responsive genes in order to activate their transcription. Second, it is known as the regulator of other transcription factors [32]. We found that the NR3C1 gene blocks two receptors (LRP6 and LRP5) placed in the cell membrane by activating SGK1 (Serine/ 


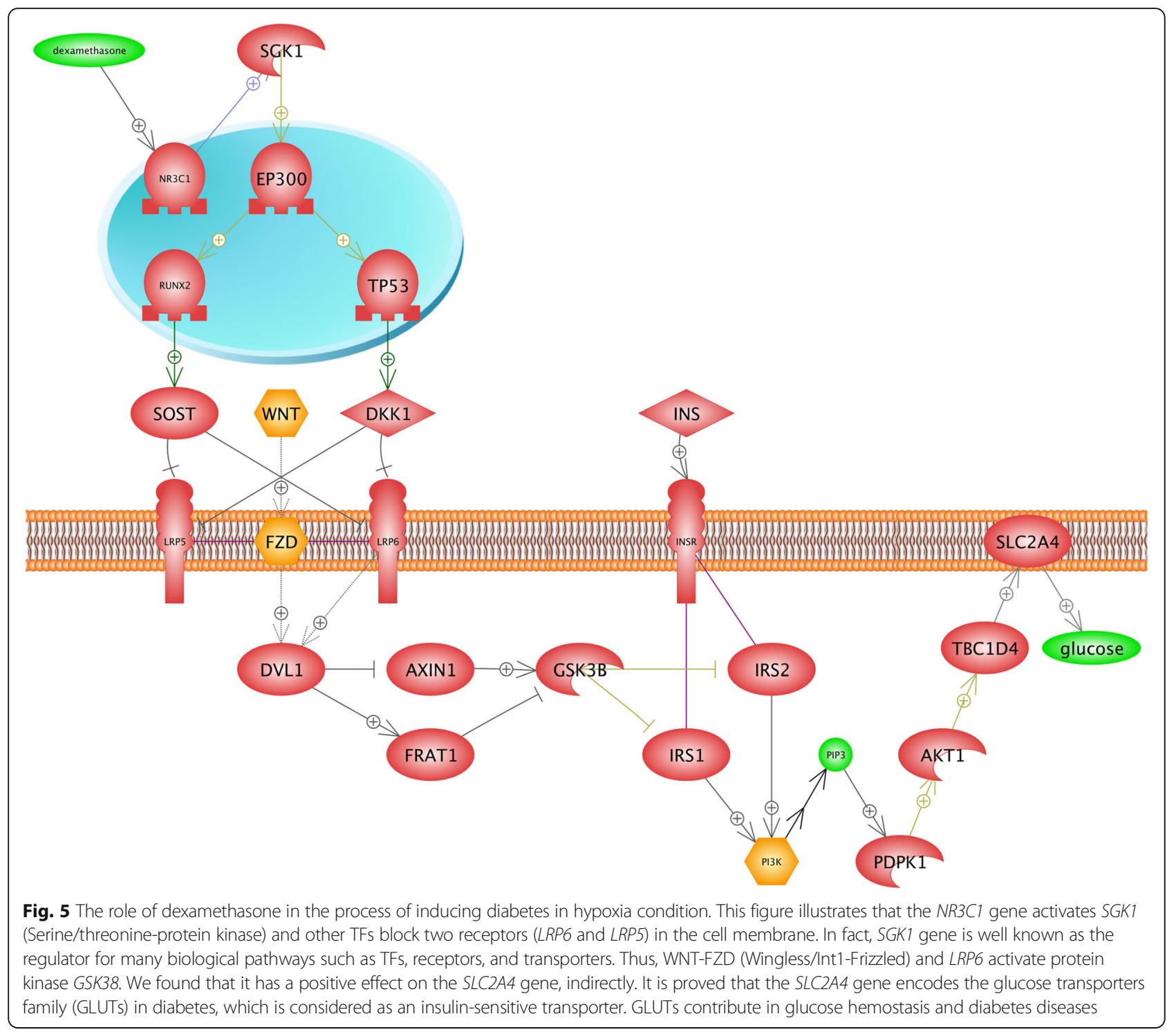

threonine-protein kinase) and other TFs. In fact, the SGK1 gene is well known to be a regulator of many biological pathways including TFs, receptors, proliferation, cellular enzymes, and transporters (Uniprot: O00141). Finally, WNT-FZD (Wingless/Int1-Frizzled), and LRP6 activate the protein kinase GSK38. These described pathways are common between diabetes and neurotoxicity. In diabetes (Fig. 5), the SLC2A4 gene encodes glucose transporters family (GLUTs), which is known as an insulin-sensitive transporter. GLUTs contribute in glucose hemostasis and diabetes diseases [99]. Figure 6 illustrates that the development of the neurotoxicity process is related to axonogenesis, neruite outgrowth, microtubule bundling, and microtubule cytoskeleton assembly. Neurotoxicity occurs when neurotoxins affect the normal activity of the nervous system and ultimately, damages the nervous tissue. In the other words, any biological process that develops the nervous system is inhibited [81]. Generally, the function of the nervous system depends on neurite (axons and dendrites) outgrowth. In the case of neurotoxicity, the number and length of neurites and axonogenes are decreased [14]. MAPT provides microtubule-associated protein tau that is found in the nervous system and is involved in the process of stabilizing microtubules, and structures the cytoskeleton [13]. Figure 5 suggests that dexamethasone inhibits the MAPT gene and decreases the growth of neurons in the nervous system, and leads to neurotoxicity. Bone resorption is observed as another side effect of dexamethasone (Fig. 7). We found that the RUNX2 (Runt-related transcription factor 2) transcription factor contributes in the osteoblast differentiation, bone resorption, and osteoclast 


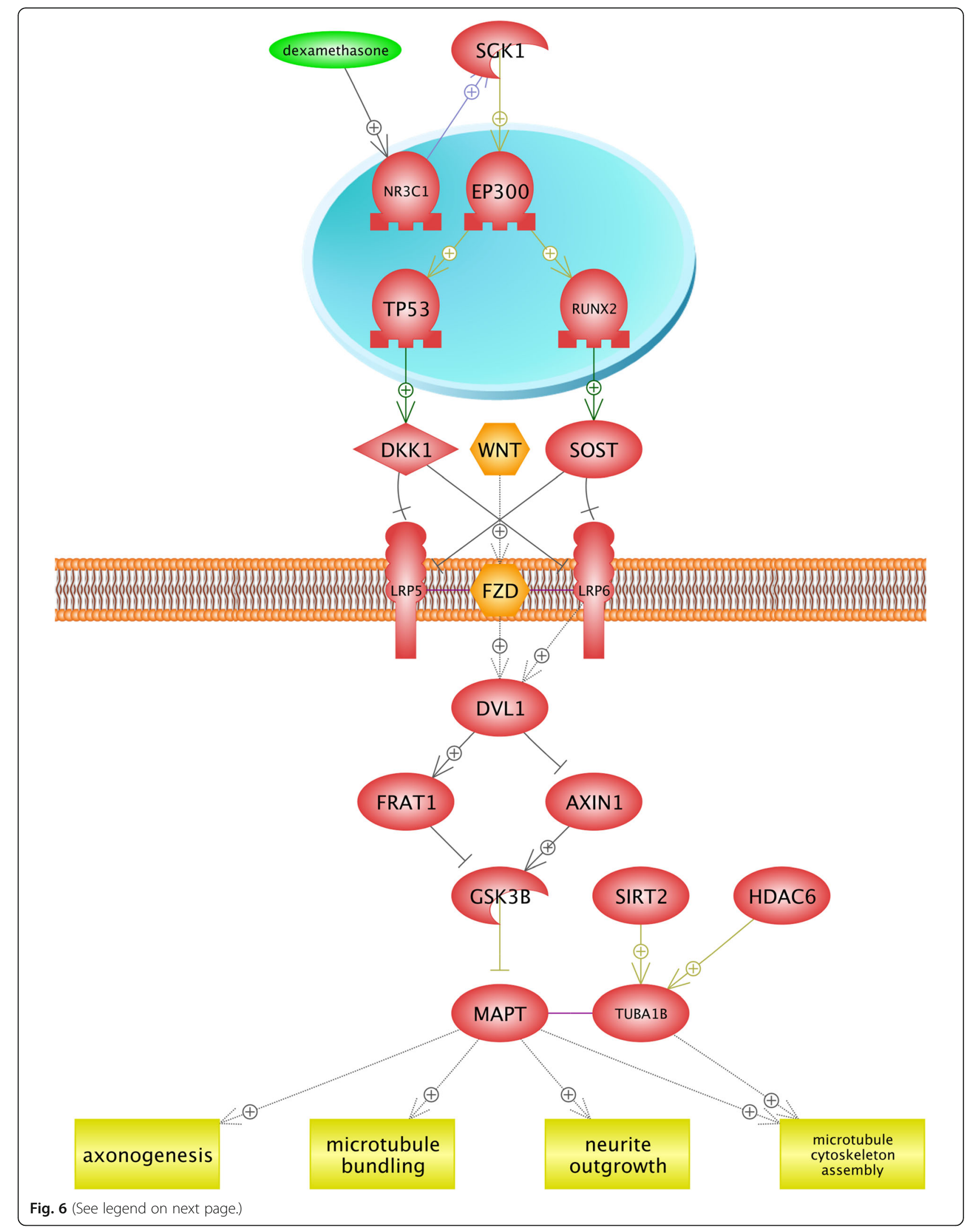


(See figure on previous page.)

Fig. 6 The development of neurotoxicity process in hypoxia condition by drug toxicity of Dexamethasone. Neurotoxicity occurs when neurotoxins affect the normal activity of the nervous system and ultimately, damage the nervous tissue. In the other words, all biological processes that lead to the development of the nervous system are inhibited. Generally, the function of the nervous system depends on neurite (axons and dendrites) outgrowth. In neurotoxicity, the number and length of neurites and axonogenes are decreased. MAPT provides microtubule-associated protein tau found in the nervous system and contributes in the process of stabilizing microtubules and structures the cytoskeleton. Figure 6 suggests that dexamethasone inhibits MAPT gene and decreases the growth of neurons in the nervous system and leads to neurotoxicity

differentiation. RUNX2 gene is the member of RUNX transcription factors, which is closely associated with the skeletal morphogenesis [10]. Figure 7 shows that RUNX2 inhibits the osteoclast differentiation by activating the tumor necrosis factor receptor superfamily (TNFRSF), while osteoclasts are considered as specialized cells that play a significant role in the bone matrix development [9]. Moreover, the RUNX2 transcription factor induces the tumor necrosis factor superfamily (TNFSF) ligand, TNFRSF, and bone resorption. It is found that TNF/TNFR molecules contribute in a variety of biological functions including the cell death, inflammation, brain function, and chemo-attractants for natural killer, monocytes, and neutrophils $[84,106]$. TNF/TNFR proteins are classified as cytokines and it is also demonstrated that cytokines might directly contribute in the bone resorptions by increasing the proliferation and activity of cells in the osteoclast lineage [8]. TNFRSF11A gene encodes the osteoprotegerin (OPG) protein, which is also known as the osteoclastogenesis inhibitory factor (OCIF). OPG and receptor activator of nuclear factor-kappaB ligand (RANKL) are classified into two main regulators of bone resorptions. In fact, many factors, including hormones and cytokines, can change the ratio of RANKL to OPG and impact on the bone resorption rate [55].

\section{Raynaud disease}

We found that hypoxia condition can lead to Raynaud disease (RD), or Raynaud's phenomenon. It is classified as a type of disorder in which the blood flow to different parts of the body, specially the fingers, are restricted by narrowing (contracting) the arteries and leads to the discoloration of fingers. It is proved that RD is related to the vasospasm (arterial spasm), and can decrease the ratio of blood flow to various organs [90]. Figure 8 shows the significance of blocking relaxation pathways. There are two factors that impact on the severity of the RD including stress and emotional upsets; therefore, it could be very beneficial to use relaxation techniques and manage stress as the alternative treatments. Our results indicate that NO (nitric oxide) and ADMA (Asymmetric dimethylarginine) are signaling pathways that contribute in Raynaud disease. ADMA is known as an endogenous analog of 1-arginine, which is metabolized to L-citrulline and dimethylamine by NG, NG-dimethylarginine dimethylaminohydrolase (DDAH). DDAH1 and DDAH2 are two isoforms of DDAH that regulate levels of blood's ADMA $[58,83]$. Moreover, $A D M A$ has a negative impact on the endothelial-NO synthase 3 (NOS3) based on the dysfunction of endothelial cells associated with a variety of diseases including coronary artery disease, diabetes mellitus, and hypertension. In fact, Endothelium refers to the interior surface of blood vessels and has a significant role in the maintenance of body's vascular structure $[83,87]$. It is also observed that the ARG2 gene (Arginase-2), which is recognized as a mitochondria enzyme, might regulate the urea cycle arginine metabolism and biosynthesis of arginine, and contributes in the process of NO production in endothelia cells. Low levels of NO production are made because of the inhibition of NOS3. It can also lead to the increase of oxygen free radical formation and endothelial dysfunction [21, 105]. Contraction was another outcome of Fig. 7. It should be noted the cold temperature is one of the important factors that can play a role in Raynaud attack. In high altitude conditions by decreasing the temperature, the body restricts blood flow to the skin to save heat body and to sustain the core body temperature. However, in this situation, the probability of constricting blood vessels will increase and it may lead to further limiting blood flow and finally Raynaud attack [68].

\section{Carcinogenesis}

We found that hypoxia conditions might contribute in the tumorigenesis such as breast cancer, ovarian cancer, and colorectal cancer. The UV radiation in highlands leads to the DNA damage in high-altitude conditions; it is also proved that there is a close association between DNA damages and cancers. Moreover, hypoxiainducible factors (HIFs) are attributed to multiple steps of tumorigenesis including tumor formation, progression, and response to therapy [79].

Results indicated that the VEGF (Vascular endothelial growth factor), known as a protein ligand, is involved in the ovarian carcinoma (OC) (Fig. 9). The VEGF gene family encodes five polypeptide growth factors including VEGF-A, VEGF-B, VEGF-C, VEGF-D, and VEGF-E, characterized by angiogenic and lymphangiogenic 


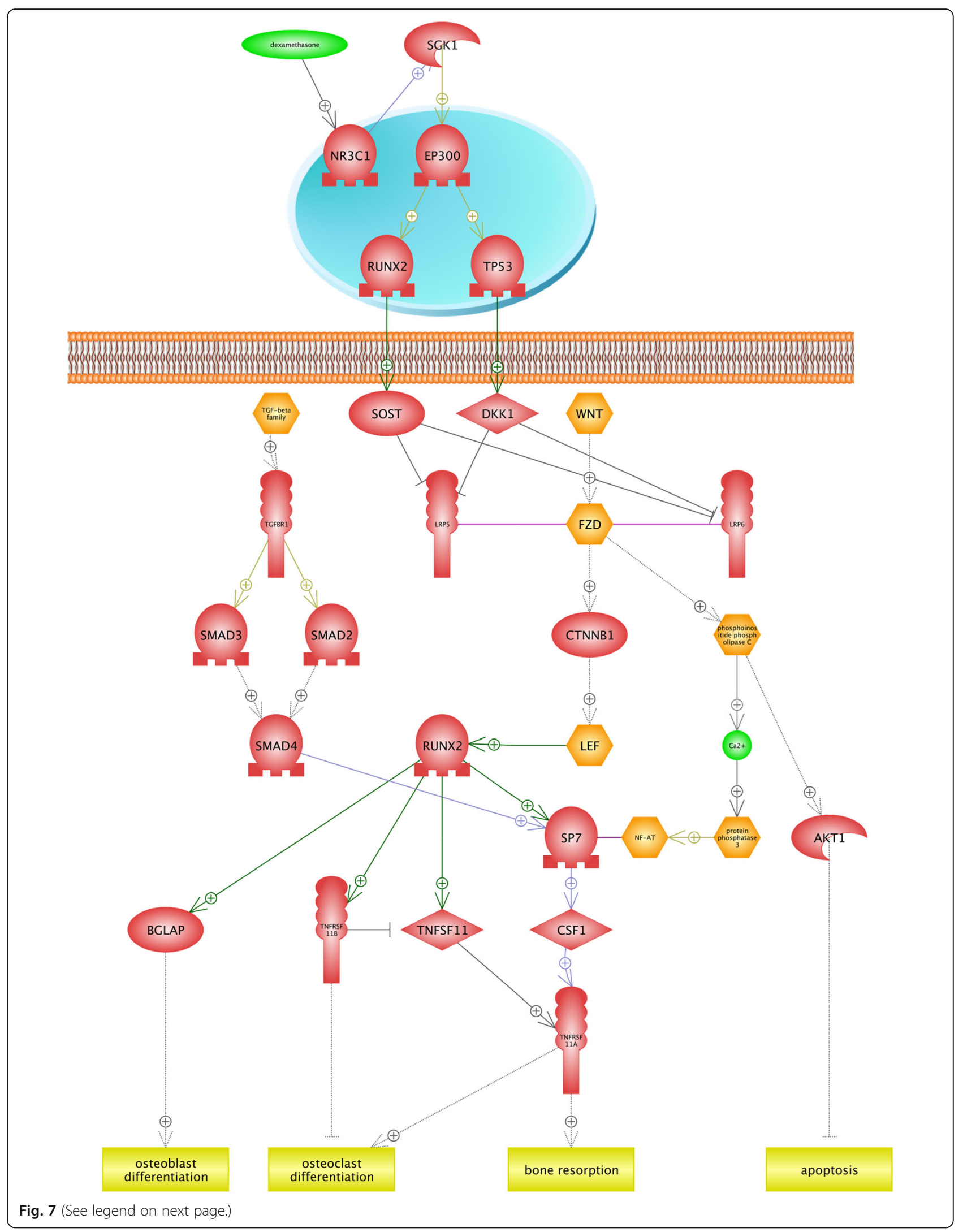


(See figure on previous page.)

Fig. 7 The association between drug toxicity of dexamethasone and bone resorption in low oxygen conditions. Bone resorption is known as another side effect of dexamethasone. According to our findings, the RUNX2 (Runt-related transcription factor 2) gene is associated with the skeletal morphogenesis by activating the tumor necrosis factor receptor superfamily (TNFRSF) that inhibits the osteoclast differentiation. Additionally, RUNX2 transcription factor induces tumor necrosis factor superfamily (TNFSF) ligand and bone resorption. TNF/TNFR proteins are classified as the cytokine, and it is also demonstrated that cytokines might contribute in bone resorptions directly by increasing proliferation and activity of cells in the osteoclast lineage. TNFRSF11A gene encodes osteoprotegerin (OPG) protein, which is also known as osteoclastogenesis inhibitory factor (OCIF)

properties in neoplasms [94]. VEGF enhances the cancer cell mobility, and also promotes the cancer cell metastasis [93]. It was observed that LRP1B, which is identified as a receptor, contributes in OC. $L R P 1 B$ gene encodes low-density lipoprotein receptor-related protein $1 \mathrm{~B}$, which plays several significant roles in the normal cell function and development due to their interactions with multiple ligands. The disruption of LRP1B is based on several types of cancers and can be considered as the tumor suppressor [62]. Deletion or downregulation of LRP1B is associated with chemotherapy resistance in $\mathrm{OC}[20]$.

Colorectal cancer (CRC) is known as the most common cancer in the world and makes $9 \%$ of all types of cancers [77]. We found that 11 candidate genes including ligands, receptors, and protein kinases are involved in CRC. Figure 10 illustrates that ATR serine/threonine kinase is related to CRC. ATR serine/threonine kinase is known as the $D N A$ damage sensor which is activated in the conditions of genotoxic stresses such as ionizing radiation, ultraviolet light, and $D N A$ replication stalling. For instance, it has a regulatory role in response to DNA double-strand breaks [6]. It is reported that mutation in ATR serine/threonine kinase leads to wide types of tumors [113].

Our gene network analysis indicated that two protein transporters including $\mathrm{APOB}$ and CLCA2 contribute in the breast cancer (Fig. 11). APOB (Apolipoprotein B) is recognized as an important member of body's lipid profile, which is an essential factor of cholesterol hemostasis [64]. The abnormal level of APOB is associated with different diseases such as cardiovascular, inflammation, and tumorigenesis [7, 30]. Furthermore, Liu et al. [63] reported that rs693 and rs1042031 polymorphisms that existed in the APOB gene increased the risk of breast cancer.

We also found that the CLCA2 gene can be considered in breast cancer. Investigations have shown the association of CLCA2 with the development of breast cancer and metastasis $[59,76]$. This protein is contained in the calcium-activated chloride channel regulator family that regulates transferring chloride across the plasma membrane. Moreover, the CLCA2 gene is known as the tumor suppressor. When the CLCA2 gene was overexpressed in negative cell lines for CLCA2, the tumorigenicity and metastasis capability of cell lines were significantly reduced [59]. It should be noted that CLCA2 plays a critical role in the epithelial differentiation of breasts and the process of promoting methylation will also lead to the downregulation of CLCA2 in breast cancers [78].

Metastasis is recognized as the main complex problem of cancer treatments and is defined as the extension of cancer cells from an initial tumor to different parts of the body $[31,43]$. Results indicate that PRKDC, CD200, $S Y T L 2$, and BDKRB2 enhanced the metastasis event (Fig. 12). PRKDC encodes DNA-PKcs protein that participates in the development of the immune system and is usually overexpressed in the cancer metastasis. Kotula et al. [56] demonstrated that there are 103 secretion proteins controlled by DNA-PKcs, and most of them are associated with metastasis. Therefore, the PRKDC gene contributes in the invasion of cancer cells by regulating secretion proteins [56]. SYTL2 (synaptotagmin like 2) is contained in the $\mathrm{C} 2$ domain-containing protein family.

It has been demonstrated that SYTL2 contributes in the ovarian cancer, and can also promote the metastatic potential in ovarian cancer when it is overexpressed [95]. Immunoglobulin superfamily (IgSF) contains two domains of extracellular and intracellular, including CD200 and CD200-R; their interaction is also involved in functions of myeloid cells [98]. The overexpression of CD200 in breast cancer can be considered as an important risk factor of metastasis [35]. The receptor of Bradykinin is encoded by the BDKRB2 gene. Bradykinin contains nine amino acid peptide chains, and is implicated in many responses including vasodilation, edema, smooth muscle spasm, and pain fiber stimulation [111].) showed that the expression of BDKRB2 is negatively associated with miR-129-1-3p in the gastric cancer, while miR-129-1-3p inhibits metastasis by targeting BDKRB2.

\section{Cardiomyopathy}

It is reported that cardiomyopathy is related to the abnormal formation of muscle heart that leads to the hypertrophic muscle. Heart muscle cells are sensitive to low oxygen concentration, which itself results in the death of cardiac myocytes [47]. Results of the current study showed that MKI67 induces cardiomyopathy, and BDKRB2 inhibits the disorder (Fig. 13). MKI67 gene encodes the nuclear protein, which is related to cellular proliferation and might also play a critical role in the chromatin organization; however, it is poorly understood 


\section{endothelial cell}

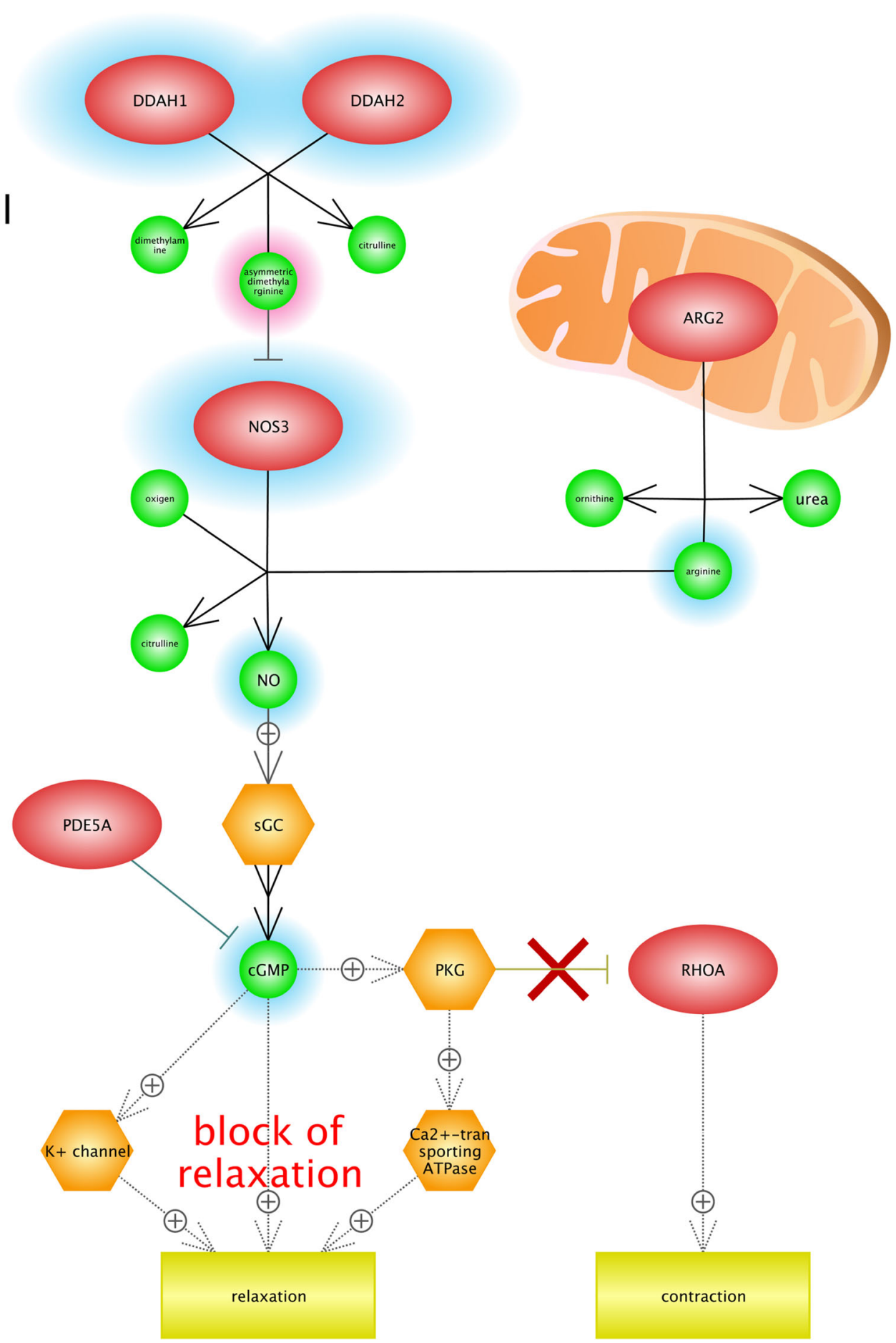

Fig. 8 Gene network analysis in order to identify the association between hypoxia condition and Raynaud disease (RD). According to our results, $\mathrm{NO}$ (nitric oxide) and ADMA (Asymmetric dimethylarginine) are considered as signaling pathways thatcontribute in RD. DDAH1 and DDAH2 are two isoforms of DDA that regulate the levels of ADMA in blood. Moreover, ADMA has a negative impact on endothelial-NO synthase 3 (NOS3) and is based on the dysfunction of endothelial cells associated with RD. It was observed that the ARG2 gene (Arginase-2), known as a

mitochondria enzyme, contributes in the process of NO production in endothelia cells. The inhibition of NOS3 will lead to the low level of NO production; it can also lead to the increase of oxygen free radical formation and endothelial dysfunction. This figure highlights the importance of relaxation pathways' block. It has been demonstrated that stress and emotional upsets impact on the severity of the RD 


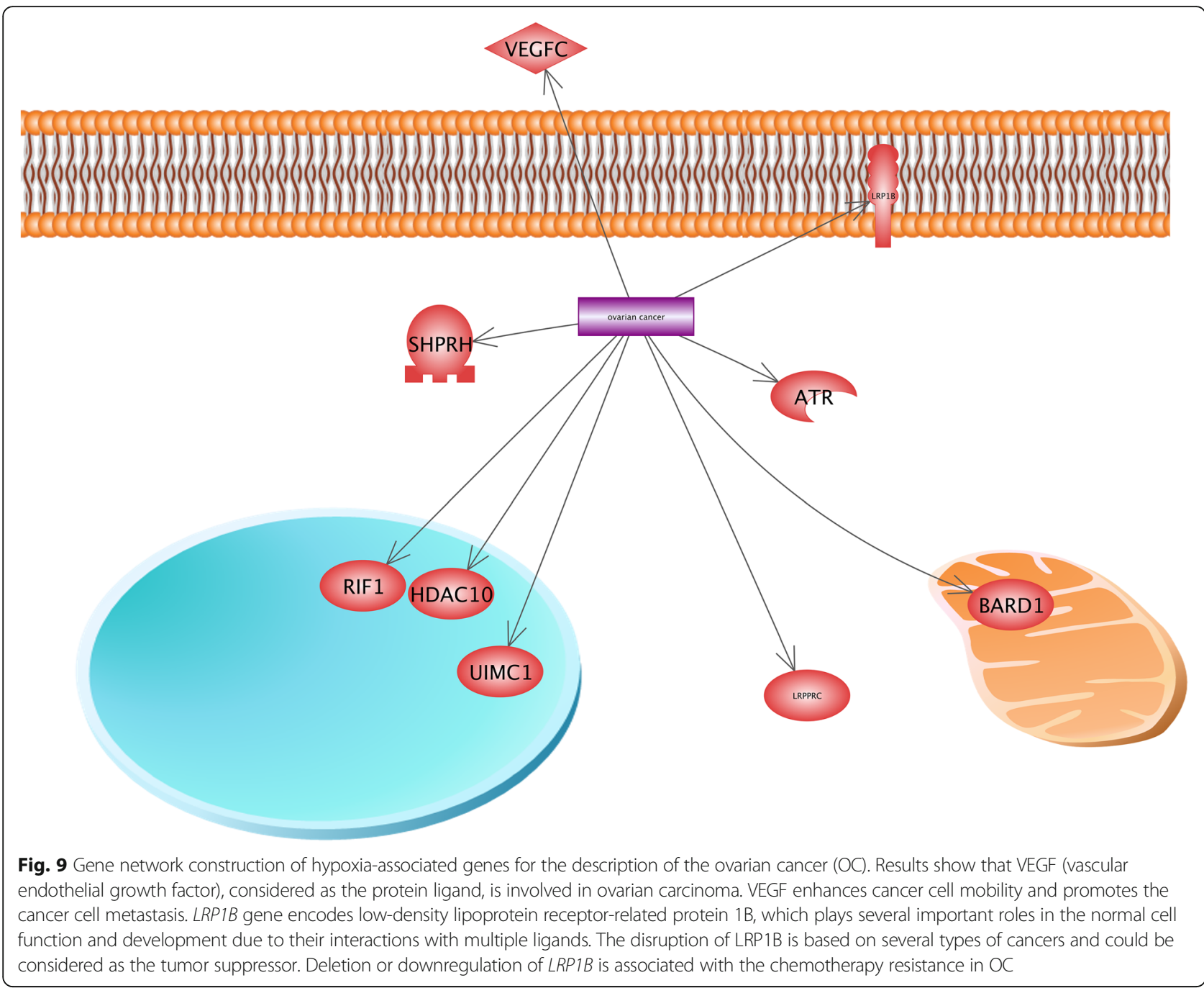

(Uniprot: KI67_HUMAN). As we described earlier, BDKRB2, known as the receptor of Bradykinin, contributes in edema, smooth muscle spasm, and pain fiber stimulation. Moreover, Gohlke et al. [33] demonstrated that Bradykinin involved in cardiac protection by blood pressure reduction, changes in renal blood flow and tubular function, inflammatory reactions. The regulatory roles of other enriched genes in cardiomyopathy networks (including SYNE2, APOB, XIRP1, ALPK3, and LRPPRC) are not clear (Fig. 13).

\section{Discussion}

Several attempts have been made to describe the association between high-altitude conditions and the incidence of diseases. However, adaptation mechanisms to highaltitude conditions are complex and include several biological pathways such as gene networks, epigenetic regulation, and different gene expression patterns. Moreover, there are irregular patterns for disorders to occur [17, $46,49]$. Therefore, we tried to identify candidate genes for diseases and explore biological pathways between hypoxia-associated genes and human diseases by gene network analysis.

High-altitude conditions can be investigated like harsh environments because there are factors including twopoint perspective, low oxygen concentration, and UV radiation. The low level of oxygen is considered as the most important problem of high-altitude conditions. Results of this study showed that several hypoxiaassociated genes are enriched in brain injury disorders including PD and AD. It is recognized as a possible explanation that the normal activity of the nervous system is extremely dependent upon normal levels of oxygen. Any disruption of oxygen consumption by brain neurons can lead to mitochondrion dysfunction and neuron necrosis. This result is in accordance with findings of Winklhofer and Haass [114] who showed that the mitochondrial dysfunction plays a central role in PD, and several PD-associated genes impact on biological pathways are associated with mitochondrial integrity. 


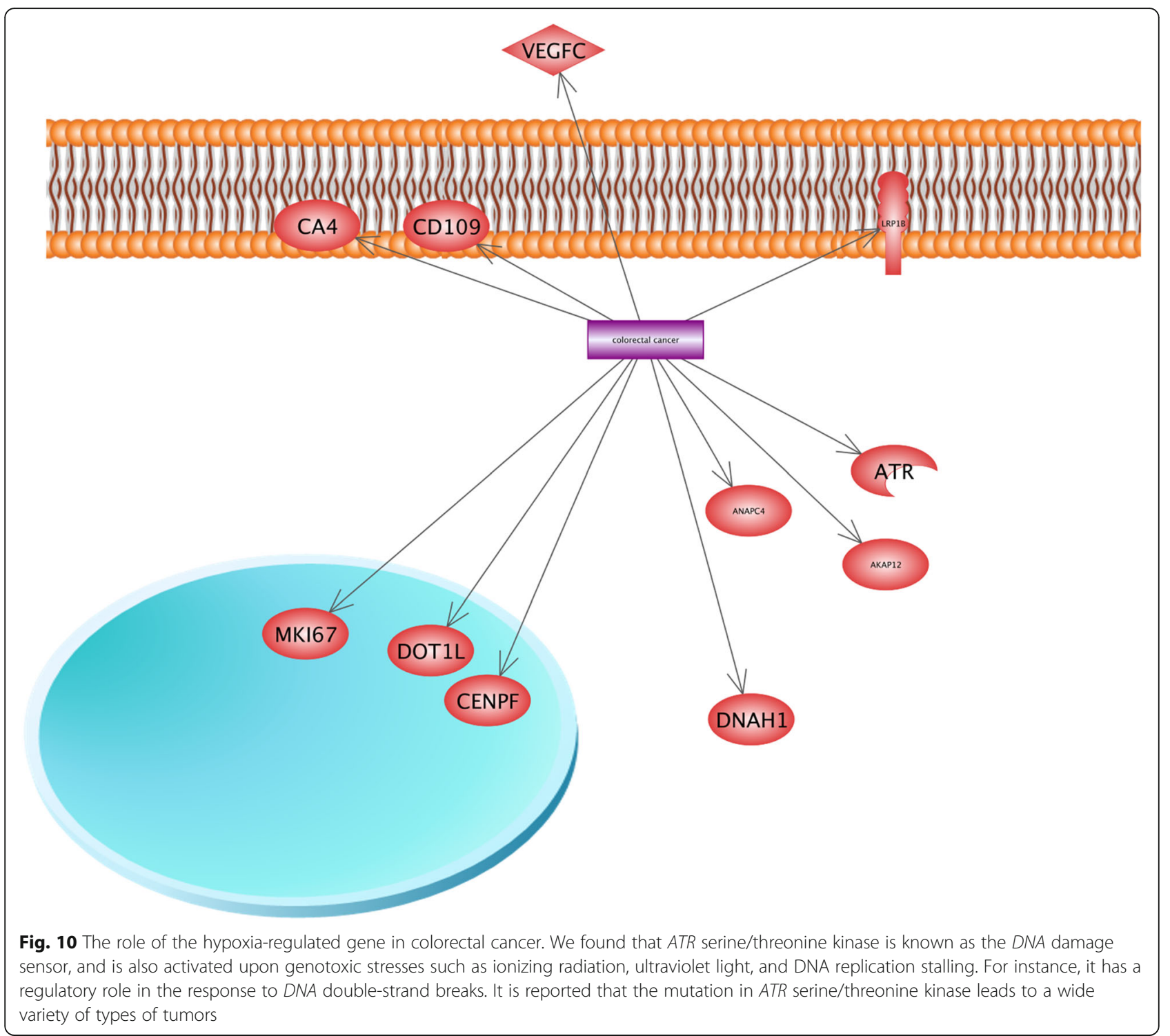

Furthermore, a strong relationship between the function of the central nervous system and supply of oxygen has been reported by Mukandala et al. [67], who indicated that the effect of low levels of oxygen is based on the release of inflammation's agents (including cytokines of neuron cells) in the early response to hypoxia that will lead to cell death. However, it was hypothesized that the inflammation response plays a protective role against brain damages. Inflammatory reactions could be having contrasting dual roles. For instance, released cytokines could improve the synaptic plasticity, modulate neuronal excitability, and also and stimulated neurogenesis and neurite outgrowth. However, a strong inflammation response in neuron cells induces overexpression or dysregulation of cytokine, which accelerates neurodegeneration [89]. It has been demonstrated, neurodegenerative disorders such as PD and
$\mathrm{AD}$ are related to the irreversible loss of structure and dysfunction of neurons $[19,54]$. Furthermore, the comparable results indicated that there is an association between the hypoxic condition and PD-related protein including $\alpha$-synuclein ( $\alpha$-Syn), and is also considered as a key protein to understand PD based on the following reasons. First $\alpha$-Syn protofibrils contributes in lewy bodies' formation and it is believed that the clump of $\alpha$-Synuclein plays a critical role in PD [118]. Second, the over-expression of $\alpha$-Syn is based on the death of neuron cells and third, the mutation in $\alpha$-Syn gene encoding is responsible for the autosomal dominant PD [54].

Another problem in high-altitude conditions is UV radiation. It is shown that high-dose UV radiation can lead to DNA damage, cell apoptosis, and tissue injury of mammals [96, 117]. Similarly, our results support 


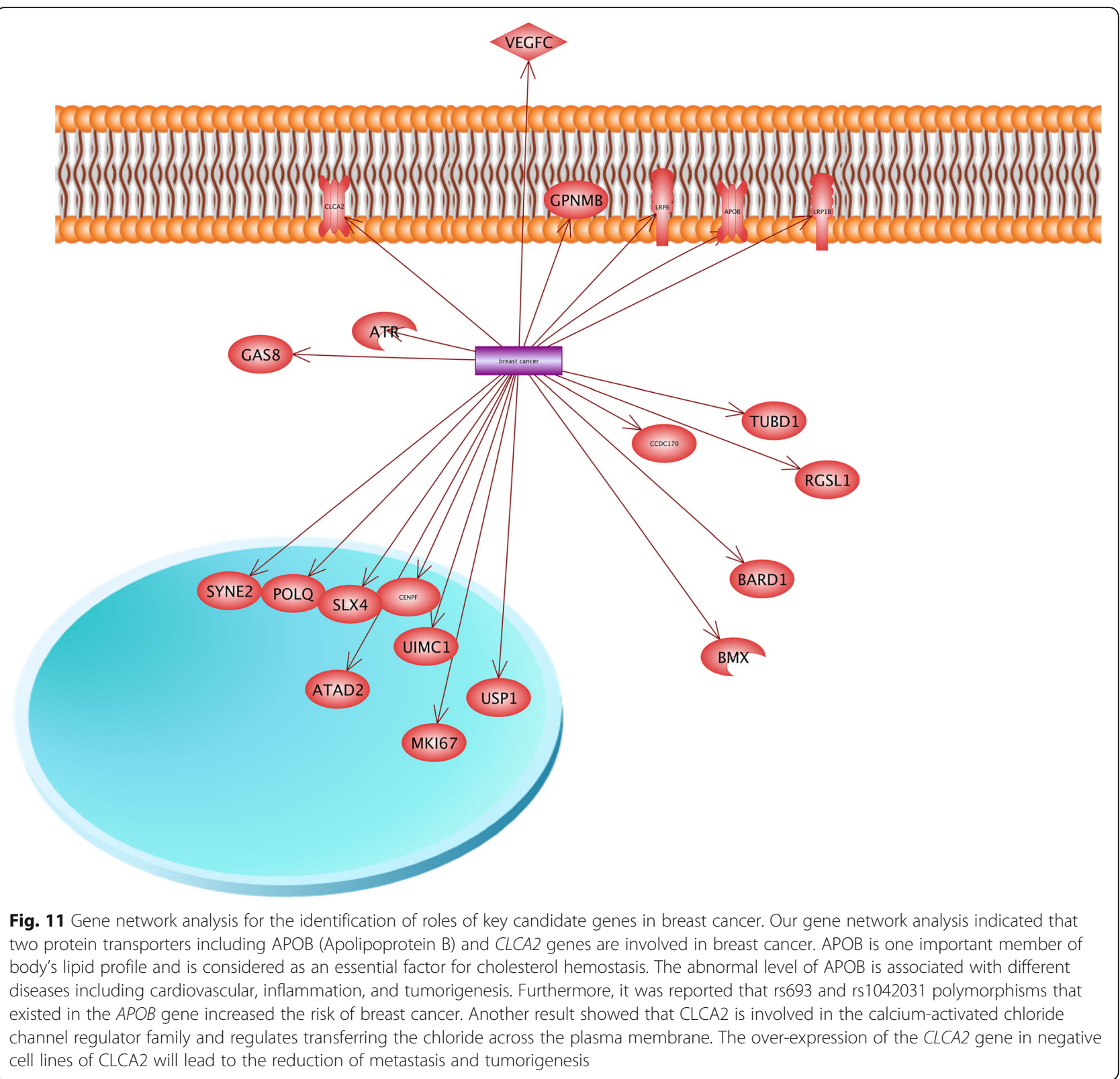

previous researches by enriching the gene associatedhypoxia in cancer networks. DNA damages are responsible for the variety of human diseases. For example, there is a close association between tumorigenesis and $D N A$ damages and consequently, numerous investigations aimed to explore the association between highaltitude and cancer mortality [12, 101, 102]. This study showed that metastasis, breast, ovarian and colorectal cancers occur in high-altitude conditions frequently. Findings of the current study are consistent with those of Hongo et al. [42] who recognized that hypoxia enhances the colon cancer through the activation of epithelial-mesenchymal transition in colon cancer cells. It is demonstrated by McEvoy et al. [65] that there is an association between hypoxia and features of cancers such as metastasis, abnormal cellular proliferation, and prevention of apoptosis. Additionally, they describe the effect of hypoxia on chemo-resistance in an ovarian cancer model.

This study indicates that many pathways of drug metabolisms are markedly altered under hypoxia conditions. Reviewing the literature, many investigations tried to describe the effect of hypoxia on pathways of drug metabolisms [45, 57, 74, 107]. There are several explanations for these results. First, for instance, many pathways of drug metabolisms such as oxidation, sulfation, and acetylation depend on the oxygen availability; therefore, the study of drug metabolism pathways in high-altitude 


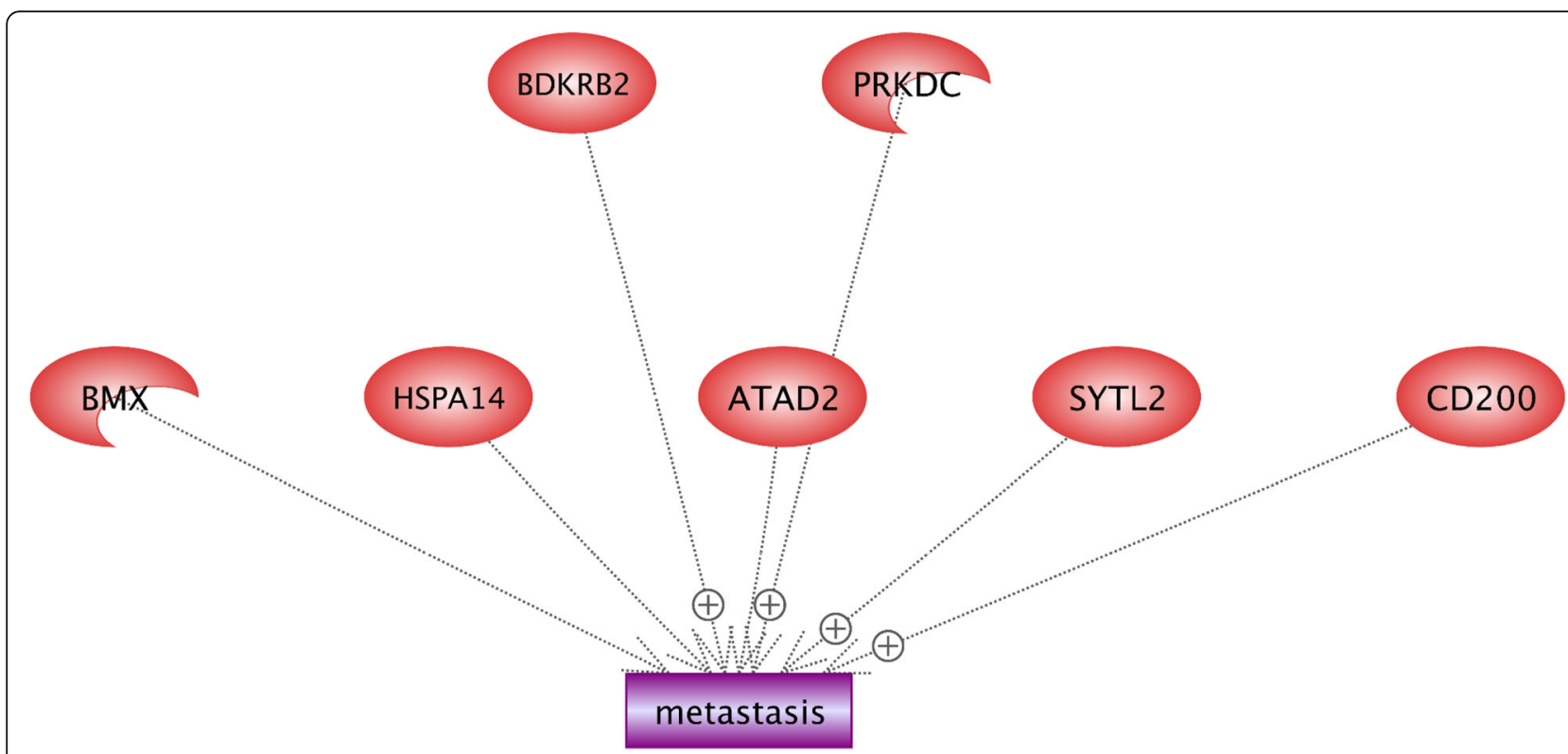

Fig. 12 Identification of regulatory roles of candidate genes in metastasis under hypoxia condition. We found that PRKDC, CD200, SYTL2, and BDKRB2 enhanced the metastasis event. PRKDC encodes DNA-PKCs protein that participates in the development of the immune system and is usually overexpressed in cancer metastasis. SYTL2 (synaptotagmin like 2) is one of the members of C2 domain-containing protein family. It is demonstrated that SYTL2 contributes in ovarian cancer and its overexpressed one can promote the metastatic potential in ovarian cancer. Immunoglobulin superfamily (IgSF) contains two domains including CD200 and CD200-R, and their interaction is also involved in functions of myeloid cells. The overexpression of CD200 in breast cancer can be considered as an important risk factor for metastasis. Bradykinin contains nine amino acid peptide chains and is implicated in many responses including vasodilation, edema, smooth muscle spasm, and pain fiber stimulation

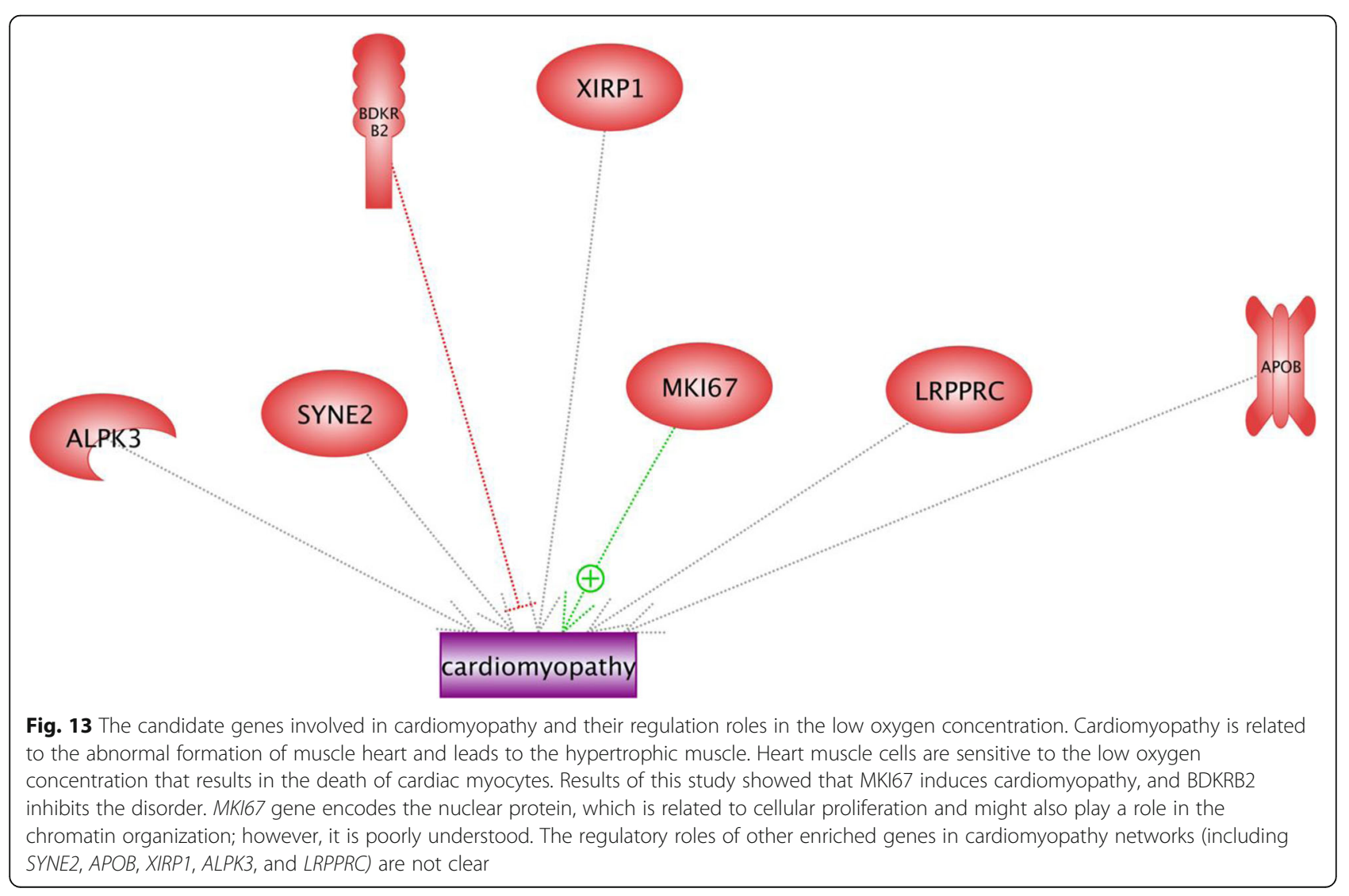


conditions can explain the side effect of drugs [57]. Second, hypoxia can impact on the pharmacokinetic features of some drugs [34]. Third, the delivery of cytotoxic drugs can be insufficient because of the negative effects of hypoxia on blood vessels and fluctuating blood flow. Thus, the concentration of cytotoxic drugs in normal tissues is higher than in cancerous tissues and will lead to the drug resistance and the destruction of normal cells. Consequently, the tumor will be resistant to chemotherapeutic treatments [115].

We found that hypoxia-associated genes can be considered in heart failure diseases. Several studies obtained evidences about the role of hypoxia in cardiomyopathy $[24,104,112]$. In addition, this study showed that MKI67 had a positive impact on cardiomyopathy. It is reported by comparable results that cardiomyocytes are able to karyokinesis (division of a cell nucleus during mitosis) in the absence of cytokinesis and are based on the increased polyploidy. Also, MKI67 proteins are expressed in the S, G1 S, and G2 phases of mitosis [80]. Furthermore, HIF expression in low oxygen conditions induced the cellular proliferation proteins such as MKI67 [5]. Considering evidences together, the polyploidy of cardiomyocytes and induced nuclear proteins of cell proliferation have significant roles in the myocardial hypertrophy disease [86]. In contrast, our results showed that $B D K R B 2$ genes inhibit cardiomyopathy. Similar results were obtained by Alves et al. [3] who showed that BDKRB2 polymorphisms are associated with cardiovascular phenotypes and suitable performance. This could be explained by the fact that the abnormal structure of $B D K R B 2$ gene is related to heart failures [60]. In addition, the existence of mutation in $B D K R B 2$ gene leads to an increase in the number of receptors of Bradykinin on the cell surface membrane and vasodilation [3].

\section{Conclusion}

The gene network analysis was carried out based on hypoxia-associated genes using an animal model in this study. We found that hypoxia-associated genes were enriched in several gene networks of disorders including Parkinson, Alzheimer, cardiomyopathy, drug toxicity, and different types of cancers. There are two main reasons that can be described for human diseases in high-altitude conditions. First, UV radiation is known as one of the most important problems in high-altitude conditions and is also responsible for a variety of human diseases. Second, low oxygen concentration influences many biological pathways, especially mitochondrion organelle. The existence of alterations in mitochondrion functions could be considered as the key symptom of human's diseases. Using the gene network analysis, this study visually presents the mechanism of disorders; it also provides new insights about key candidate genes of diseases.

\section{Abbreviations}

AD: Alzheimer's disease; ADMA: Asymmetric dimethylarginine; APP: Amyloid precursor protein; APOB: Apolipoprotein B; ARG2: Arginase-2; A $\beta$ : $\beta$-amyloid peptide; BDKRB2: Bradykinin receptor B2; CD200: Cluster of Differentiation 200; CLCA2: Chloride channel Accessory 2; CYCS: Cytochrome C; CRC: Colorectal cancer; DDAH: Dimethylarginine dimethylaminohydrolase; DNM1L: Dynamin 1 Like; GLUTs: Glucose transporters family; HIF1A: Hypoxiainducible factor 1-alpha; IgSF: Immunoglobulin superfamily; LRP: LDL Receptor Related Protein; MCU: Mitochondrial calcium uniporter; MKI67: Marker of Proliferation Ki-67; MPTP: Mitochondrial permeability transition pore; NADH: Nicotinamide-adenine dinucleotide; NOS: Nitric oxide synthase 1; NR3C1: Nuclear receptor subfamily 3 group $C$ member 1; OC: Ovarian carcinoma; OCIF: Osteoclastogenesis inhibitory factor; OPA1: Optic atrophy 1; OPG: Osteoprotegerin; PD: Parkinson disease; PRKD C: Protein Kinase, DNAActivated; RANKL: Receptor activator of nuclear factorkappaB ligand; RD: Raynaud disease; ROS: Reactive oxygen species; RUNX2: Runt-related transcription factor 2; SGK1: Serine/threonine-protein kinase; SLC2A4: Solute carrier family 2 member 4; SOD2: Superoxide Dismutase-2; a-Syn or SNCA: a-synuclein; SYTL2: Synaptotagmin like 2; TOM: Translocase of the outer mitochondrial membrane; TNFSF: Tumor necrosis factor superfamily; TNFRSF: Tumor necrosis factor receptor superfamily; UV: Ultraviolet; VEGF: Vascular endothelial growth factor; WNTFZD: Wingless/Int1-Frizzled

\section{Supplementary Information}

The online version contains supplementary material available at https://doi. org/10.1186/s43141-021-00184-5.

Additional file 1. Supplementary material

Additional file 2: Table S1. The list of selected hypoxia- associated genes for gene network analysis. Table S2: Principle component analysis of phenotypic traits for discriminate analysis and clustering the highland and lowland populations. Table S3: The classification of highland and lowland chickens based on the discriminate analysis.

\section{Acknowledgements \\ Not applicable}

\section{Authors' contributions}

HKK, MD, and AE conceived the study. HKK performed the experiments and prepared the first draft of the manuscript. RT revised the manuscript. HKK, EE, and AN contributed in data analyses. The authors read and approved the final manuscript.

\section{Funding}

This study was funded by Iran National Science Foundation (INSF; Grant number: 99013627) and it was also supported by Shahid Bahonar University of Kerman.

\section{Availability of data and materials \\ The genome resequencing reads were deposited in the browser of chickenSD database (http://bigd.big.ac.cn/chickensd/)}

\section{Declarations}

\section{Ethics approval and consent to participate}

This investigation is in accordance with relevant guidelines and regulations of Shiraz University. All experimental protocols were approved by the Institute of Biotechnology at Shiraz University. The reference number is not applicable

Consent for publication

Not applicable

Competing interests

The authors declare that they have no competing interests. 


\section{Author details}

${ }^{1}$ Institute of Biotechnology, Shiraz University, Shiraz, Iran. ${ }^{2}$ Department of Animal Science, Faculty of Agriculture, Shahid Bahonar University of Kerman, Kerman, Iran. ${ }^{3}$ School of Animal and Veterinary Sciences, The University of Adelaide, Adelaide, Australia. ${ }^{4}$ Genomics Research Platform, School of Life Sciences, La Trobe University, Melbourne, Victoria, Australia. ${ }^{5}$ Department of Animal Science, School of Agriculture, Shiraz University, Shiraz, Iran. ${ }^{6}$ Institute of Animal Husbandry, Inner Mongolia Academy of Agricultural \& Animal Husbandry Sciences, Hohhot 010031, China.

\section{Received: 15 January 2021 Accepted: 21 May 2021}

\section{Published online: 08 July 2021}

\section{References}

1. Alavi MV, Fuhrmann N (2013) Dominant optic atrophy, OPA1, and mitochondrial quality control: understanding mitochondrial network dynamics. Mol Neurodegener 8, 32. https://doi.org/10.1186/1750-1326-8-32.

2. Arenas E, Saltó C, Villaescusa C (2015) WNT signaling in midbrain dopaminergic neuron development and cell replacement therapies for Parkinson's disease. SpringerPlus 4:L49

3. Alves CR et al (2013) Vascular reactivity and ACE activity response to exercise training are modulated by the $+9 /-9$ bradykinin B2 receptor gene functional polymorphism. Physiol Genomics 45:487-492

4. Beitz JM (2014) Parkinson's disease: a review. Front Biosci 6:65-74

5. Bekeredjian R et al (2010) Conditional HIF-1a expression produces a reversible cardiomyopathy. PloS One 5:e11693

6. Blackford AN, Jackson SP (2017) ATM, ATR, and DNA-PK: the trinity at the heart of the DNA damage response. Mol Cell 66:801-817

7. Borgquist $S$ et al (2016) Apolipoproteins, lipids and risk of cancer. Int $J$ Cancer 138:2648-2656

8. Boyce B (2013) Advances in the regulation of osteoclasts and osteoclast functions. J Dent Res 92:860-867

9. Boyle WJ, Simonet WS, Lacey DL (2003) Osteoclast differentiation and activation. Nature 423:337

10. Bruderer M, Richards R, Alini M, Stoddart MJ (2014) Role and regulation of RUNX2 in osteogenesis. Eur Cell Mater 28:269-286

11. Burt DW (2007) Emergence of the chicken as a model organism: implications for agriculture and biology. Poultry Sci 86:1460-1471

12. Burtscher M (2014) Effects of living at higher altitudes on mortality: a narrative review. Aging Dis 5:274

13. Caillet-Boudin ML, Buée L, Sergeant N, Lefebvre B (2015) Regulation of human MAPT gene expression. Mol Neurodegener 10:28

14. Ceresa $C$ et al (2014) Characterization of and protection from neurotoxicity induced by oxaliplatin, bortezomib and epothilone-B. Anticancer Res 34 517-523

15. Cha MY, Kim DK, Mook-Jung I (2015) The role of mitochondrial DNA mutation on neurodegenerative diseases. Exp Mol Med 47:e150

16. Chakraborty C, Hsu CH, Wen ZH, Lin CS, Agoramoorthy G (2009) Zebrafish: a complete animal model for in vivo drug discovery and development. Currt Drug Metab 10:116-124

17. Chavala MA (2018) A journey between high altitude hypoxia and critical patient hypoxia: What can it teach us about compression and the management of critical disease? Med Intensiva (Engl Ed) 42(6):380-390

18. Cogburn LA, Porter TE, Duclos MJ, Simon J, Burgess SC, Zhu JJ, Cheng HH, Dodgson JB, Burnside J (2007) Functional Genomics of the Chicken A Model Organism. Poult Sci 86(10):2059-2094

19. Cookson MR (2009) a-Synuclein and neuronal cell death. Mol Neurodegener 4,9

20. Cowin PA et al (2012) LRP1B deletion in high-grade serous ovarian cancers is associated with acquired chemotherapy resistance to liposomal doxorubicin. Cancer Res 72:4060-4073

21. de Haro Miralles J et al (2009) Nitric oxide: link between endothelial dysfunction and inflammation in patients with peripheral arterial disease of the lower limbs. Interact Cardiov Tho 9:107-112

22. Doan $\mathrm{R}$ et al (2012) Whole-genome sequencing and genetic variant analysis of a Quarter Horse mare. BMC Genomics. 13. https://doi.org/10.1186/14 71-2164-13-78

23. Dias M et al (2017) SNP detection using RNA-sequences of candidate genes associated with puberty in cattle. Genet Mol Res 16. https://doi.org/10.4238/ gmr16019522
24. Dimitiru L, Dimitiru A, Stamatin M (2012) 1156 Hypoxic Perinatal Cardiomyopathy-Diagnosis and Evolution. Arch Dis Child 97:A331-A331

25. Dodgson JB, Romanov MN (2004) Use of chicken models for the analysis of human disease. Current Protocols in. Hum Genet 40:15.5. 1-15.5. 12

26. Eales K, Hollinshead K, Tennant D (2016) Hypoxia and metabolic adaptation of cancer cells. Oncogenesis 5:e190

27. Ebrahimie E, Moussavi-Nik S-H, Newman M, Lardelli M (2016) A zebrafish homologue of the Alzheimer's disease-associated PRESENILIN isoform PS2V regulates inflammatory and other responses to hypoxic stress. J Alzheimers Dis 52:581-608

28. Esfandiari $P$, Dadpasand M, Kharrati-Koopaee $H$, Atashi H, Gharghi A, Niazi A (2020) Bioinformatics, phylogenetic and variant association analysis of Ovocalyxin-32 gene reveals its contribution to egg production traits in native chickens. Animal Gene 17:200108

29. Esteras $\mathrm{N}$ et al (2013) Calmodulin levels in blood cells as a potential biomarker of Alzheimer's disease. Alzheimers Res Ther 5:55

30. Fouchier SW (2005) et al., High frequency of APOB gene mutations causing familial hypobetalipoproteinaemia in patients of Dutch and Spanish descent. J Med Genet 42:e23-e23

31. Geiger TR, Peeper DS (2009) Metastasis mechanisms. Biochimica et Biophysica Acta (BBA)-Reviews on. Cancer 1796:293-308

32. Giarraputo $J$ et al (2016) Medical morbidities and DNA methylation of NR3C1 in preterm infants. Pediatr Res 81:68

33. Gohlke P, Tschöpe C, Unger T (1997) Bradykinin and cardiac protection. Hypertens Heart:159-172

34. Gong W et al (2017) Effect of hypoxia on the pharmacokinetics and metabolism of zaleplon as a probe of CYP3A1/2 activity. RSC Adv 7 : 25414-25421

35. Gorczynski RM, Clark DA, Erin N, Khatri I (2011) Role of CD200 expression in regulation of metastasis of EMT6 tumor cells in mice. Breast Cancer Res $\mathrm{Tr}$ 130:49-60

36. Grisham M, Jourd'Heuil D, Wink D (2000) chronic inflammation and reactive oxygen and nitrogen metabolism-implications in DNA damage and mutagenesis. Aliment Pharm Ther 14:3-9

37. Haining RL, Achat-Mendes C (2017) Neuromelanin, one of the most overlooked molecules in modern medicine, is not a spectator. Neural Regen Res 12:372

38. Halestrap AP (2009) What is the mitochondrial permeability transition pore? J Mol Cell Cardiol 46:821-831

39. Harbauer AB, Zahedi RP, Sickmann A, Pfanner N, Meisinger C (2014) The protein imports machinery of mitochondria - a regulatory hub in metabolism, stress, and disease. Cell Metab 19:357-372

40. Hewitt VL, Whitworth AJ (2017) Mechanisms of Parkinson's disease: Lessons from Drosophila. Curr Top Dev Biol 121:173-200

41. Hinkelbein J et al (2017) Thirty Minutes of Hypobaric Hypoxia Provokes Alterations of Immune Response, Haemostasis, and Metabolism Proteins in Human Serum. Int J Mol Sci 18:1882

42. Hongo $\mathrm{K}$ et al (2013) Hypoxia enhances colon cancer migration and invasion through promotion of epithelial-mesenchymal transition. J Surg Res 182:75-84

43. Hunter KW, Crawford NP, Alsarraj J (2008) Mechanisms of metastasis. Breast Cancer Res 10:S2

44. JBonda D et al (2011) The mitochondrial dynamics of Alzheimer's disease and Parkinson's disease offer important opportunities for therapeutic intervention. Curr Pharm design 17:3374-3380

45. Jones DP, Aw TY, Shan X (1989) Drug metabolism and toxicity during hypoxia. Drug Metab Rev 20:247-260

46. Julian CG (2017) Epigenomics and human adaptation to high altitude. Journal of Applied Physiology 123(5):1362-1370

47. Julian $\mathrm{R}$ (2007) The response of the heart and pulmonary arteries to hypoxia, pressure, and volume. A short review. Poultry Sci 86:1006-1011

48. Kann O, Schuchmann S, Buchheim K, Heinemann U (2003) Coupling of neuronal activity and mitochondrial metabolism as revealed by NAD (P) H fluorescence signals in organotypic hippocampal slice cultures of the rat. Neurosci 119:87-100

49. Ke J, Wang L, Xiao D (2017) Cardiovascular adaptation to high-altitude hypoxia. Hypoxia and human diseases 117

50. Kerr JB (2005) Understanding the factors that affect surface ultraviolet radiation. Opt Eng 44(4):041002

51. Kharrati-Koopaee H, Ebrahimie E, Dadpasand M, Niazi A, Esmailizadeh A (2019) Genomic analysis reveals variant association with high altitude adaptation in native chickens. Sci Rep. https://doi.org/10.1038/s41598-019-45661-7 
52. Khurana P, Sugadev R, Jain J, Singh SB (2013) HypoxiaDB: a database of hypoxia-regulated proteins. Database. https://doi.org/10.1093/database/bat074

53. Khurana P, Tiwari D, Sugadev R, Sarkar S, Singh SB (2016) A comprehensive assessment of networks and pathways of hypoxia-associated proteins and identification of responsive protein modules. Netw Model Anal Health Inform Bioinform 5:17

54. Kim T, Vemuganti R (2017) Mechanisms of Parkinson's disease-related proteins in mediating secondary brain damage after cerebral ischemia. J Cereb Blood Flow Metab 37:1910-1926

55. Kostenuik PJ (2005) Osteoprotegerin and RANKL regulate bone resorption, density, geometry and strength. Curr Opin Pharmacol 5:618-625

56. Kotula E et al (2015) DNA-PKcs plays role in cancer metastasis through regulation of secreted proteins involved in migration and invasion. Cell Cycle 14:1961-1972

57. Lee K, Roth RA, LaPres JJ (2007) Hypoxia, drug therapy and toxicity. Pharmacol Therapeut 113:229-246

58. Lee $\mathrm{W}$ et al (2018) Asymmetric dimethylarginine (ADMA) is identified as a potential biomarker of insulin resistance in skeletal muscle. Sci Rep 8:2133

59. Li X, Cowell JK, Sossey-Alaoui K (2004) CLCA2 tumour suppressor gene in $1 p 31$ is epigenetically regulated in breast cancer. Oncogene 23:1474

60. Li $Y$ et al (2012) Bradykinin $\beta 2$ receptor- 58 T/C gene polymorphism and essential hypertension: a meta-analysis. Plos One 7:e43068

61. Ling N (2003) Rotenoneôa review of its toxicity and use for fisheries management

62. Liu CX, Li Y (2001) Obermoeller-McCormick, L. M., Schwartz, A. L., Bu, G. The putative tumor suppressor LRP1B, a novel member of the low density lipoprotein (LDL) receptor family, exhibits both overlapping and distinct properties with the LDL receptor-related protein. J Biol Chem 276, 2888928896

63. Liu X et al (2013) Associations of polymorphisms of rs693 and rs1042031 in apolipoprotein B gene with risk of breast cancer in Chinese. Jpn J Clin Oncol 43:362-368

64. Ma MZ, Yuan SQ, Chen YM, Zhou ZW (2018) Preoperative apolipoprotein B/ apolipoprotein a1 ratio: a novel prognostic factor for gastric cancer. OncoTarget Ther 11:2169

65. McEvoy LM et al (2015) Identifying novel hypoxia-associated markers of chemoresistance in ovarian cancer. BMC Cancer 15:547

66. Michiels C (2004) Physiological and pathological responses to hypoxia. Ame J Pathol 164:1875-1882

67. Mukandala G, Tynan R, Lanigan S, O'Connor JJ (2016) The effects of hypoxia and inflammation on synaptic signaling in the CNS. Brain Sci $6: 6$

68. Musa R, Qurie A (2020 Jan) Raynaud Disease. [Updated 2020 Nov 19]. In: StatPearls. StatPearls Publishing, Treasure Island (FL) Available from: https:// www.ncbi.n/m.nih.gov/books/NBK499833

69. Mortazavi A, Williams BA, McCue K, Schaeffer L, Wold B (2008) Mapping and quantifying mammalian transcriptomes by RNA-Seq. Nat Methods. 5:621-628

70. Moussavi-Nik SH et al (2015) Alzheimer's disease-related peptide PS2V plays ancient, conserved roles in stimulation of ã-secretase activity and suppression of the unfolded protein response under hypoxia. Human Mol Genet 24:3662-3678

71. Muz B, de la Puente P, Azab F, Azab AK (2015) The role of hypoxia in cancer progression, angiogenesis, metastasis, and resistance to therapy. J Alzheimer Dis 46(3):553-569

72. O'Day DH, Eshak K, Myre MA (2015) Calmodulin binding proteins and Alzheimer's disease. J Alzheimer Dis 46:553-569

73. O'Brien RJ, Wong PC (2011) Amyloid precursor protein processing and Alzheimer's disease. Annu Rev Neurosci 34:185-204

74. Papadopoulou MV, Ji M, Bloomer WD (2011) Hypoxia-Dependent Retina Toxicity of NLCQ-1 (NSC 709257) in BALB/c Mice. Comparison with Tirapazamine. Basic Clin Pharmacol 108:396-399

75. Pashaiasl M, Ebrahimi M, Ebrahimie E (2016) Identification of the key regulating genes of diminished ovarian reserve (DOR) by network and gene ontology analysis. Mol Biol Reports. 43:923-937

76. Qiang YY LCZ, Sun R, Zheng LS, Peng LX, Yang JP, Qian CN (2018) Along with its favorable prognostic role, CLCA2 inhibits growth and metastasis of nasopharyngeal carcinoma cells via inhibition of FAK/ERK signaling. J Exp Clin Cancer Res 37(1):1-14

77. Rafiemanesh H et al (2016) Colorectal cancer in Iran: Epidemiology and morphology trends. EXCLI J 15:738

78. Ramena G, Yin Y, Yu Y, Walia V, Elble RC (2016) CLCA2 interactor EVA1 is required for mammary epithelial cell differentiation. PLoS One 11:e0147489
79. Rankin E, Giaccia A (2008) The role of hypoxia-inducible factors in tumorigenesis. Cell Death Differ 15:678

80. Richardson GD, Laval S, Owens WA (2015) Cardiomyocyte regeneration in the mdx mouse model of nonischemic cardiomyopathy. Stem Cells Dev 24 1672-1679

81. Robinette BL, Harrill JA, Mundy WR, Shafer TJ (2011) In vitro assessment of developmental neurotoxicity: use of microelectrode arrays to measure functional changes in neuronal network ontogeny1. Front Neuroeng 4:1

82. Schito L, Semenza GL (2016) Hypoxia-inducible factors: master regulators of cancer progression. Trends Cancer 2:758-770

83. Sciacqua A, Grillo N, Quero M, Sesti G, Perticone F (2012) Asymmetric dimethylarginine plasma levels and endothelial function in newly diagnosed type 2 diabetic patients. Int J Mol Sci 13:13804-13815

84. Sedger LM, McDermott MF (2014) TNF and TNF-receptors: from mediators of cell death and inflammation to therapeutic giants-past, present and future. Cytokine Growth Factor Rev 25:453-472

85. Shariepour Z, Aliakbari BA (2011) The effects of cloudiness and total ozone on UV-B radiation in Esfahan region. Geophy J 5(4):74-88

86. Shlyakhto E et al (2007) Cellular aspects of pathogenesis of hypertrophic cardiomyopathy: Role of cardiomyocyte polyploidy and activation of nuclear antigen of the proliferating cell in myocardium. Cell Tissue Biol 1:582-588

87. Sibal LC, Agarwal SD, Home PH, Boger R (2010) The role of asymmetric dimethylarginine (ADMA) in endothelial dysfunction and cardiovascular disease. Curr Cardiol Rev 6:82-90

88. Simon F et al (2004) Hydroxyl radical activation of a Ca2+-sensitive nonselective cation channel involved in epithelial cell necrosis. Am J of Physiol-Cell Ph 287:C963-C970

89. Sochocka M, Diniz BS, Leszek J (2017) Inflammatory response in the CNS: friend or foe? Mol Neurobiol 54(10):8071-8089

90. Solomons HD (2011) Raynaud's phenomenon. Cardiovascular Journal of Africa 22:233

91. Stepień K, Dzierzega-Lecznar A, Tam I (2007) The role of neuromelanin in Parkinson's disease--new concepts. Wiad Lek 60:563-569

92. Stevenson JW et al (2016) The amyloid precursor protein of Alzheimer's disease clusters at the organelle/microtubule interface on organelles that bind microtubules in an ATP dependent manner. PloS One 11:e0147808

93. Su JL et al (2006) The VEGF-C/Flt-4 axis promotes invasion and metastasis of cancer cells. Cancer Cell 9:209-223

94. Su JL et al (2007) The role of the VEGF-CNEGFR-3 axis in cancer progression. Brit J Cancer 96:541

95. Sung HY, Han J, Ju W, Ahn JH (2016) Synaptotagmin-like protein 2 gene promotes the metastatic potential in ovarian cancer. Oncology Rep 36:535-541

96. Svobodová AR et al (2012) DNA damage after acute exposure of mice skin to physiological doses of UVB and UVA light. Arch Dermatol Res 304:407-412

97. Takeuchi T, Watanabe Y, Takano-Shimizu T, Kondo S (2006) Roles of jumonji and jumonji family genes in chromatin regulation and development. Dev Dyn 235:2449-2459

98. Talebian F, Bai XF (2012) The role of tumor expression of CD200 in tumor formation, metastasis and susceptibility to $T$ lymphocyte adoptive transfer therapy. Oncoimmunology 1:971-973

99. Tarazona-Santos E et al (2010) Diversity in the glucose transporter-4 gene (SLC2A4) in Humans reflects the action of natural selection along the oldworld primates' evolution. PloS One 5:e9827

100. Terraneo $L$ et al (2017) Brain adaptation to hypoxia and hyperoxia in mice. Redox Biol 11:12-20

101. Thiersch M, Swenson E, Haider T, Gassmann M (2017) Reduced cancer mortality at high altitude: The role of glucose, lipids, iron and physical activity. Exp Cell Res 356:209-216

102. Thiersch M, Swenson ER (2018) High altitude and cancer mortality. High Alt Med Biol 19(2):116-23

103. Thompson R, Buttigieg J, Zhang M, Nurse C (2007) A rotenone-sensitive site and $\mathrm{H}_{2} \mathrm{O} 2$ are key components of hypoxia-sensing in neonatal rat adrenomedullary chromaffin cells. Neurosci 145:130-141

104. Tintu A et al (2009) Hypoxia induces dilated cardiomyopathy in the chick embryo: mechanism, intervention, and long-term consequences. PloS One 4:e5155

105. Tousoulis D, Kampoli AM, Tentolouris Nikolaos Papageorgiou C, Stefanadis C (2012) The role of nitric oxide on endothelial function. Curr Vasc Pharmacol 10:4-18

106. Twohig JP, Cuff SM, Yong AA, Wang EC (2011) The role of tumor necrosis factor receptor superfamily members in mammalian brain development, function and homeostasis. Rev Neurosci 22:509-533 
107. Urner M et al (2012) Effect of hypoxia and dexamethasone on inflammation and ion transporter function in pulmonary cells. Clin Exp Immunol 169:119-128

108. Vasiev B, Balter A, Chaplain M, Glazier JA, Weijer CJ (2010) Modeling gastrulation in the chick embryo: formation of the primitive streak. PLoS One 5:e10571

109. Wakabayashi K, Tanji K, Mori F, Takahashi H (2007) The Lewy body in Parkinson's disease: Molecules implicated in the formation and degradation of a-synuclein aggregates. Neuropathology 27:494-506

110. Wang D, Luo L, Guo J (2014a) miR-129-1-3p inhibits cell migration by targeting BDKRB2 in gastric cancer. Med Oncol 31:98

111. Wang RS, Oldham WM, Loscalzo J (2014b) Network-based association of hypoxia-responsive genes with cardiovascular diseases. New J Phys 16: 105014

112. Watanabe Y, Kusuoka H, Fukuchi K, Fujiwara T, Nishimura T (1997) Contribution of hypoxia to the development of cardiomyopathy in hamsters. Cardiovasc Res 35:217-222

113. Weber AM, Ryan AJ (2015) ATM and ATR as therapeutic targets in cancer. Pharmacol Therapeut 149:124-138

114. Winklhofer KF, Haass C (2010) Mitochondrial dysfunction in Parkinson's disease. Biochimica et Biophysica Acta (BBA). Mol Basis Dis 1802:29-44

115. Wouters A, Pauwels B, Lardon F, Vermorken JB (2007) Implications of in vitro research on the effect of radiotherapy and chemotherapy under hypoxic conditions. Oncologist 12:690-712

116. Yan MH, Wang X, Zhu X (2013) Mitochondrial defects and oxidative stress in Alzheimer disease and Parkinson disease. Free Radical Bio Med 62:90-101

117. Yel M, Güven T, Türker H (2014) Effects of ultraviolet radiation on the stratum corneum of skin in mole rats. J Radiat Res Appl Sci 7:506-511

118. Zhang J, Darley-Usmar V (2012) Mitochondrial dysfunction in neurodegenerative disease: Protein aggregation, autophagy, and oxidative stress. In: Mitochondrial dysfunction in neurodegenerative disorders Springer, London, pp 95-111.

119. Zhang K et al (2018) Investigation of hypoxia networks in ovarian cancer via bioinformatics analysis. J Ovarian Res 11:16

120. Zucca FA et al (2017) Interactions of iron, dopamine and neuromelanin pathways in brain aging and Parkinson's disease. Prog Neurobiol 155:96-119

\section{Publisher's Note}

Springer Nature remains neutral with regard to jurisdictional claims in published maps and institutional affiliations.

\section{Submit your manuscript to a SpringerOpen ${ }^{\circ}$ journal and benefit from:}

- Convenient online submission

- Rigorous peer review

- Open access: articles freely available online

High visibility within the field

- Retaining the copyright to your article

Submit your next manuscript at $\boldsymbol{\nabla}$ springeropen.com 